\title{
Depression after Subarachnoid Hemorrhage: A Systematic Review
}

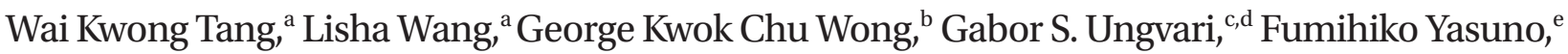 \\ Kelvin K.F. Tsoi, ${ }^{\mathrm{f}, \mathrm{g}}$ Jong S. Kim ${ }^{\mathrm{h}}$ \\ aDepartment of Psychiatry, Chinese University of Hong Kong, Hong Kong, China \\ ${ }^{b}$ Department of Surgery, Chinese University of Hong Kong, Hong Kong, China \\ 'University of Notre Dame Australia, Fremantle, Australia \\ ${ }^{d}$ Division of Psychiatry, Medical School, University of Western Australia, Crawley, Australia \\ 'Department of Psychiatry, National Center for Geriatrics and Gerontology, Obu, Japan \\ fJockey Club School of Public Health and Primary Care, Chinese University of Hong Kong, Hong Kong, China \\ ${ }^{9}$ Stanley Ho Big Data Decision Analytics Research Centre, Chinese University of Hong Kong, Hong Kong, China \\ hDepartment of Neurology, Asan Medical Center, University of Ulsan College of Medicine, Seoul, Korea
}

Background and Purpose Depression is common and debilitating illness accompanying many neurological disorders including non-traumatic subarachnoid hemorrhage (SAH). The aim of this systematic review was to identify and critically appraise all published studies that have reported the frequency, severity and time course of depression after SAH, the factors associated with its development and the impact of depression on patients' quality of life after SAH.

Methods The PubMed database was searched for studies published in English that recruited at least 40 patients ( $>18$ years old) after SAH who were also diagnosed with depression.

Results Altogether 55 studies covering 6,327 patients met study entry criteria. The frequency of depression ranged from 0\% to 61.7\%, with a weighted proportion of 28.1\%. Depression remained common even several years after the index SAH. Depression after SAH was associated with female sex, premorbid depression, anxiety, substance use disorders or any psychiatric disorders, and coping styles. Comorbid cognitive impairment, fatigue, and physical disability also increased the risk of depression. Aneurysmal SAH and infarction may be related to depression as well. Depression reduces the quality of life and life satisfaction in patients after SAH.

Conclusions Depression is common after SAH and seems to persist. Further research is needed to clarify its time course and identify the neuroendocrine and neurochemical factors and brain circuits associated with the development of post-SAH depression. Randomized controlled treatment trials targeting $\mathrm{SAH}-$ related depression are warranted.

Keywords Subarachnoid hemorrhage; Depression; Systematic review
Correspondence: Wai Kwong Tang Department of Psychiatry, Shatin Hospital, Chinese University of Hong Kong, Shatin, N.T., Hong Kong, China Tel: $+852-2636-7760$

Fax: +852-2647-5321

E-mail: tangwk@cuhk.edu.hk

Received: August 9, 2019 Revised: December 18, 2019 Accepted: January 8, 2020

\section{Introduction}

Subarachnoid hemorrhage $(\mathrm{SAH})$ is a relatively uncommon and severe type of stroke. As patients are affected by SAH at a mean age of 55 years, they can lose many years of productive life. The rupture of an intracranial aneurysm is the underlying cause in $85 \%$ of SAH cases. ${ }^{1}$ Approximately 55\% of patients survive SAH and regain independent functioning, whereas 19\% 
remain dependent and 26\% die. ${ }^{1}$ Many survivors of SAH have long-term deficits in cognition, and decreased quality of life. ${ }^{2}$ Neuropsychiatric disturbances such as depression, anxiety, post-traumatic stress disorder, and fatigue are not uncommon, yet often neglected in patients with $\mathrm{SAH}{ }^{3}$

Depression is common in patients with neurological diseases such as Alzheimer's disease, Parkinson's disease, traumatic brain injury and stroke. ${ }^{4}$ Depression is a frequent consequence of head injury, affecting up to $61 \%$ of patients. Depression is associated with worse global outcomes, impaired social functioning, difficulty performing activities of daily living, and a lower quality of life. ${ }^{5}$

Post-stroke depression contributes to disability and increased mortality following stroke. Depression is increasingly becoming a standard part of post-stroke assessment and rehabilitation. ${ }^{6}$ However, there is still a lack of methodically sound psychopharmacological and psychosocial treatment trials on SAH-related depression.

The aims of this systematic review were as follows: (1) to determine the frequency, severity and time course of depression after SAH; (2) to identify the factors associated with the development of depression after $\mathrm{SAH}$, including patients' demographic data, baseline characteristics of SAH, psychological factors including anxiety and cognitive impairment, somatic complications related to SAH (neuroendocrine changes, infarcts, and preexisting and post-SAH medical comorbidities); and (3) to evaluate the impact of depression on patients' quality of life following SAH.

\section{Methods}

\section{Literature search}

This review was conducted according to the Preferred Reporting Items for Systematic Reviews and Meta-Analyses (PRISMA) guideline. The principal author (W.K.T.) searched the PubMed, EMBASE, PsycINFO, and Ovid Nursing databases on December 5, 2018, using the keywords "depression" or "mood" or "depressive" and "subarachnoid." Two authors (W.K.T. and L.W.) read every title and abstract, obtained the full texts of potentially relevant papers, and applied inclusion and exclusion criteria to each text. Any uncertainties were discussed. W.K.T. also scrutinized the reference lists of included papers to identify further studies.

\section{Inclusion criteria}

Studies were included in the review if they (1) were written in English, (2) were published in peer-reviewed journals, (3) included 10 or more patients who had survived non-traumatic SAH and were older than 18 years, and (4) assessed patients for depression using a validated single or multiple item self-re- port instrument or diagnostic interview.

\section{Exclusion criteria}

Publications were excluded if they were (1) case reports, (2) pediatric studies (patients $<18$ years old), (3) dissertations, or (4) articles with no primary data (reviews, editorials letters, etc.), (5) sample size $<40$, (6) poor quality (a Strengthening the Report of Observational Studies in Epidemiology [STROBE] checklist score $\leq 13$, i.e., $60 \%$ of maximum score).

\section{Data extraction}

Two authors (W.K.T. and L.W.) independently extracted the following data from the studies included in the review: study characteristics (aims/objectives, study design, inclusion and exclusion criteria, criteria for and measurement of depression), participants' characteristics (definition of the study population, age, gender, number, ethnicity, and socio-economic status of the patients at the beginning and end of the study, the number of deaths due to $\mathrm{SAH}$, drop-outs, and patients lost to follow-up before the end of study, first or recurrent SAH, severity of SAH, comorbidities and complications) and results (characteristics of patients' subgroups, outcome data, and relationship between depression and patients' characteristics or SAH characteristics and/or outcomes).

\section{Quality assessment}

We used STROBE statement for quality assessment of the included papers. ${ }^{8}$ It consists of 22 items. We scored each item 1 point. The maximum possible score is 22 .

\section{Data synthesis}

Statistical analyses were performed in Software R (package metaphor \& meta, R Foundation for Statistical Computing, Vienna, Austria). The results are presented as a narrative review and are also tabulated. Frist, the weighted proportion of the frequency of depression was calculated. We conducted a metaanalysis of frequency of depression, using the variance-stablizing double-arscine method transformation. ${ }^{9}$ Pooled estimates in both the overall (and subgroup) analyses were calculated using the Hartung-Knapp-Sidik-Jonkman method, under the random effect model. ${ }^{10}$ Statistical heterogeneity among the trials was assessed, and $P<0.1$ was considered as statistical significance." Level of heterogeneity was assessed by $I^{2}$, which describes the percentage of total variation across studies because of heterogeneity rather than chance alone. A randomeffects model for the trials with statistically significant heterogeneity was used. Subgroup analyses were performed according to data collection settings, i.e., interview and questionnaire. 
Second we conducted a meta-analysis of frequency of depression, using the variance-stablizing double-arscine method transformation. ${ }^{9}$ Pooled estimates in both the overall (and subgroup) analyses were calculated using the Hartung-Knapp-Sidik-Jonkman method, under the random effect model. ${ }^{10}$ Publication bias was examined by Funnel plot and Egger's regression test. Data from the identical cohorts was reported only once. Where depression was assessed with more than one method in a study, only the results with the most commonly used assessment method were considered. Where data from two or more time points after SAH were available, data from the earlier time point was included in the analysis.

\section{Results}

The electronic search identified 2,383 publications potentially eligible for the review. One hundred and six full texts were retrieved for detailed evaluation, of which 51 studies were excluded (Figure 1). Fifty-five studies covering 6,327 patients (range, 40 to 1,181) in 47 cohorts (data from five cohorts were used in 13 publications ${ }^{12-24}$ ) met the inclusion criteria (Table 1). The majority of studies (46 of 55) included more female than men. Quality of included studies was very different and the STROBE score varied from 14 to 22 (Supplementary Table 1).

Fifty-one of 55 studies (93\%) used one of the following screening or rating scales to ascertain the presence of depression: Hospital Anxiety Depression Scale (HADS), ${ }^{18,19,25-40}$ Beck Depression Inventory (BDI), ${ }^{12-21,40-51}$ Center for Epidemiologic Studies Depression (CESD), ${ }^{22-24,52,53}$ Montgomery Åsberg Depression Rating Scale (MADRS), ${ }^{54,55}$ Geriatric Depression Scale (GDS) ${ }_{1}^{56,57}$ International Classification of Diseases 10th Edition
(ICD-10)-Symptom-Rating questionnaire, ${ }^{58}$ Neuropsychiatric Inventory, ${ }^{3}$ Emotional State Questionnaire, ${ }^{59}$ Psychological General Well-Being Index, ${ }_{1}^{60}$ and the Zung Depression Scale (ZDS). ${ }^{61}$ Less commonly used measures of depression included the Structural Clinical Interview for Diagnostic and Statistical Manual of Mental Disorders Fourth Edition (DSM-IV) axis I disorders, $_{1}{ }^{62}$ clinical interview ${ }_{1}^{63}$ Cornell personality questionnaire, ${ }_{1}^{64}$ and Depression Skala ${ }^{65}$ (Table 1).

Twenty-seven studies recruited patients early after SAH and assessed them for depression later. Twenty-six studies recruited patients and collected data at one or more defined time points after SAH (range, 4 days to 10 years) (Table 1).

\section{Frequency, severity, and time course of depression after SAH}

\section{Frequency of depression after SAH}

Thirty-seven studies $(n=5,340)$ reported the frequency of depression after $\mathrm{SAH}$. The frequency ranged widely depending on the assessment method and how long it was performed after the index SAH.

The frequency of depression reported in the 35 studies $(n=5,217)$ that applied rating instruments/questionnaires was in the range of $0 \%$ to $61.7 \%$, with a weighted frequency of $28.1 \%$. Depression was assessed at 4 days to 10.1 years after ictus (Table 2). The weighted frequency of depression at $\leq 1$ and $>1$ year after ictus were $32.2 \%$ and $27.3 \%$, respectively. The frequency in the two studies $(n=123)$ that used interviews to diagnose depression found 20\% to 25\% (weighted proportion $=21.6 \%$ ) of patients depressed (Table 2). ${ }^{62,63}$

In the meta-analysis, the overall pooled frequency of depres-

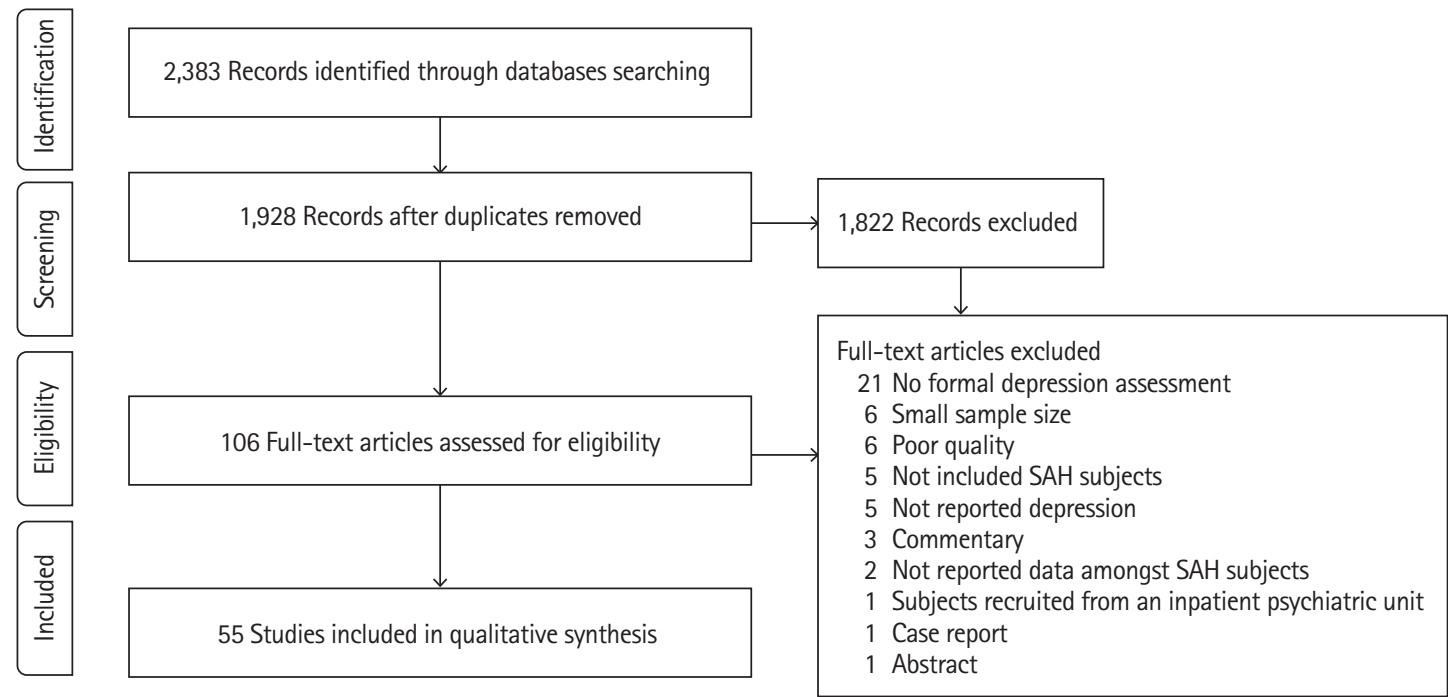

Figure 1. Flow-chart diagram presenting the selection of eligible studies. SAH, subarachnoid hemorrhage. 
Table 1. Characteristics of the studies included in the systematic review

\begin{tabular}{|c|c|c|c|c|c|c|c|}
\hline \multirow[b]{2}{*}{ Study } & \multirow[b]{2}{*}{ Design } & \multicolumn{3}{|c|}{ Participants } & \multirow[b]{2}{*}{$\begin{array}{l}\text { Aetiology of } \\
\text { SAH }\end{array}$} & \multirow[b]{2}{*}{ Timing after SAH } & \multirow[b]{2}{*}{$\begin{array}{c}\text { Tool used to diagnose } \\
\text { depression }\end{array}$} \\
\hline & & No. & Age $($ mean $\pm S D)$ & $\begin{array}{l}\text { Male sex } \\
(\%)\end{array}$ & & & \\
\hline Ljunggren et al. (1985) ${ }^{63}$ & Cross & 40 & NR & 42.5 & Aneu & $40-45 \mathrm{mo}$ & Interview \\
\hline Hütter et al. $(1995)^{20,21}$ & Cross & 58 & 46 (median) & 43 & Aneu & $1-5 y r$ & BDI \\
\hline Hellawell et al. (1999) $)^{39}$ & Long & 44 & $49.7 \pm 14.6$ & 45 & Aneu & $\begin{array}{c}6 \mathrm{mo}(\mathrm{T} 1) \\
1 \mathrm{yr}(\mathrm{T} 2) \\
2 \mathrm{yr}(\mathrm{T} 3)\end{array}$ & HADS \\
\hline Fertl et al. (1999) $)^{51}$ & Cross & 40 & 51 (SD NR) & 40 & Aneu & $\begin{array}{l}\text { Average } 22 \text { mo } \\
\text { (range, NR) }\end{array}$ & $\mathrm{BDI}$ \\
\hline Carter et al. $(2000)^{61}$ & Cross & 182 & 52 (SD NR) & NR & Aneu & $1-5 \mathrm{yr}$ & ZDS \\
\hline $\begin{array}{l}\text { Powell et al. }(2002)^{18} \text { and } \\
\text { Powell et al. }(2004)^{19}\end{array}$ & Long & 52 & $46.9 \pm 10.4$ & 33 & Aneu & $\begin{array}{c}3 \mathrm{mo}(\mathrm{T} 1) \\
9 \mathrm{mo}(\mathrm{T} 2) \\
18 \mathrm{mo}(\mathrm{T} 3)\end{array}$ & $B D I \& H A D S$ \\
\hline Fontanella et al. $(2003)^{50}$ & Long & 37 & $55.3 \pm 8.8$ & 35 & Aneu & $6 \mathrm{mo}$ & BDI \\
\hline Bellebaum et al. (2004) ${ }^{49}$ & Long & 32 & $54.4 \pm 12.5$ & NR & Aneu & $23-28 \mathrm{mo}$ & $\mathrm{BDI}$ \\
\hline Morris et al. $(2004)^{40}$ & Cross & 70 & $45.2 \pm 15.2$ & 39 & Aneu (81\%) & $14-23 \mathrm{mo}$ & BDI \& HADS \\
\hline Salmond et al. (2006) ${ }^{48}$ & Cross & 20 & $58.6 \pm 2.1$ & 20 & Aneu & 14-99 mo & $\mathrm{BDI}$ \\
\hline Wermer et al. $(2007)^{38}$ & Cross & 610 & $54.3 \pm 9.0$ & 36 & Aneu & $2.3-18.8 \mathrm{yr}$ & HADS \\
\hline Kreitschmann-Andermahr et al. $(2007)^{47}$ & Cross & 40 & 43.8 (SD NR) & NR & Aneu & $12-66 \mathrm{mo}$ & BDI \\
\hline Preiss et al. $(2007)^{46}$ & Long & 75 & 45.7 (SD NR) & 33 & Aneu & $1 \mathrm{yr}$ & $\mathrm{BDI}$ \\
\hline Orbo et al. $(2008)^{45}$ & Long & 42 & 48 (SD NR) & 40.4 & Aneu & $1 \mathrm{yr}$ & BDI \\
\hline Visser-Meily et al. (2009) ${ }^{36}$ & Cross & 141 & $54.1 \pm 12.3$ & 33.3 & Aneu & $2-4 \mathrm{yr}$ & HADS \\
\hline Haug et al. (2009) ${ }^{55}$ & Long & 46 & 53 (median) & 37 & Aneu & $1 \mathrm{yr}$ & MADRS \\
\hline King et al. (2009) $)^{37}$ & Long & 178 & $54.7 \pm 12.6$ & 26 & Aneu & NR & HADS \\
\hline Mukerji et al. (2010) 35 & Cross & 77 & $54.3 \pm 10.8$ & 32.5 & Aneu (84\%) & $12-266 \mathrm{mo}$ & HADS \\
\hline $\begin{array}{l}\text { Passier et al. }(2010)^{14} \text {, Passier et al. } \\
(2011)^{15} \text {, Passier et al. }(2012)^{16}\end{array}$ & Long & 111 & $52.8 \pm 13.0$ & 18 & Aneu & $3 \mathrm{mo}$ & BDI \\
\hline Meyer et al. $(2010)^{44}$ & Long & 113 & $54.4 \pm 14.1$ & 32.7 & Aneu & $\begin{array}{l}\text { At discharge (T1) } \\
12 \mathrm{mo}(\mathrm{T} 2)\end{array}$ & BDI \\
\hline Caeiro et al. $(2011)^{54}$ & Long & 108 & $53.5 \pm 14.2$ & 30 & Aneu (56\%) & $\leq 4$ day & MADRS \\
\hline Alfieri et al. (2008) $)^{17}$ & Long & 38 & $44.3 \pm 13.7$ & 42 & Aneu (47\%) & $\begin{array}{c}\text { On admission (T1) } \\
1 \text { mo (T2) } \\
1 \text { yr (T3) } \\
3 \text { yr (T4) } \\
5 \text { yr (T5) }\end{array}$ & BDI \\
\hline Hedlund et al. $(2011)^{62}$ & Long & 83 & $52 \pm 9$ & 36 & NR & $\begin{array}{l}10 \text { day (T1) } \\
7 \text { mo (T2) }\end{array}$ & $\begin{array}{l}\text { Structured clinical interview for } \\
\text { DSM-IV axis I disorders }\end{array}$ \\
\hline Wong et al. $(2012)^{57}$ & Long & 90 & $45 \pm 11$ & 76 & Aneu & $3 \mathrm{mo}$ & GDS \\
\hline Latimer et al. $(2013)^{33}$ & Cross & 23 & 52.7 (SD NR) & NR & Aneu & $40-45 \mathrm{mo}$ & HADS \\
\hline von Vogelsang et al. $(2013)^{34}$ & Cross & 217 & $50.6 \pm 12$ & 29 & Aneu & $8.8-12 y r$ & HADS \\
\hline Kreiter et al. $(2013)^{53}$ & Long & 216 & $51.2 \pm 13.8$ & 36 & Aneu (87\%) & $\begin{array}{c}3 \mathrm{mo}(\mathrm{T} 1) \\
1 \mathrm{yr}(\mathrm{T} 2)\end{array}$ & CESD \\
\hline Wong et al. $(2013)^{56}$ & Long & 120 & 51 (median) & 68 & Aneu & $1 \mathrm{yr}$ & GDS \\
\hline Vetkas et al. (2013) ${ }^{59}$ & Cross & 114 & $54 \pm 13$ & 32 & Aneu & $1-10 \mathrm{yr}$ & Emotional State Questionnaire \\
\hline Noble et al. $(2014)^{32}$ & Cross & 414 & 44.6 (SD NR) & 25 & Aneu (68.4\%) & $0-34 \mathrm{yr}$ & HADS \\
\hline Wong et al. (2014) & Cross & 103 & $55 \pm 10$ & 29 & Aneu & $1-4 \mathrm{yr}$ & Neuropsychiatric inventory \\
\hline Gill et al. $(2015)^{31}$ & Cross & 93 & $49.67 \pm 10.05$ & 20.43 & NR & $2-58 \mathrm{mo}$ & HADS \\
\hline Hütter et al. (2014) ${ }^{43}$ & Cross & 45 & $47.1 \pm 10.7$ & 44 & Aneu (64\%) & $3-5 y r$ & $\mathrm{BDI}$ \\
\hline $\begin{array}{l}\text { Boerboom et al. }(2014)^{22} \text { and } \\
\text { Boerboom et al. }(2016)^{23,24}\end{array}$ & Long & 76 & $53.8 \pm 11.5$ & 31.6 & Aneu & $\begin{array}{l}0.4 \mathrm{yr}(\mathrm{T} 1) \\
3.9 \mathrm{yr}(\mathrm{T} 2)\end{array}$ & CESD \\
\hline
\end{tabular}


Table 1. Continued

\begin{tabular}{|c|c|c|c|c|c|c|c|}
\hline \multirow[b]{2}{*}{ Study } & \multirow[b]{2}{*}{ Design } & \multicolumn{3}{|c|}{ Participants } & \multirow[b]{2}{*}{$\begin{array}{l}\text { Aetiology of } \\
\text { SAH }\end{array}$} & \multirow[b]{2}{*}{ Timing after SAH } & \multirow[b]{2}{*}{$\begin{array}{l}\text { Tool used to diagnose } \\
\text { depression }\end{array}$} \\
\hline & & No. & Age $($ mean \pm SD) & $\begin{array}{c}\text { Male sex } \\
(\%)\end{array}$ & & & \\
\hline von Vogelsang et al. $(2015)^{29}$ & Long & 88 & $52.6 \pm 14.2$ & 34.1 & Aneu & $\begin{array}{c}6 \mathrm{mo}(\mathrm{T} 1) \\
1 \mathrm{yr}(\mathrm{T} 2) \\
2 \mathrm{yr}(\mathrm{T} 3)\end{array}$ & HADS \\
\hline Buunk et al. (2015) ${ }^{30}$ & Cross & 200 & 58.7 (SD NR) & 36.5 & Aneu & $2-10 y r$ & HADS \\
\hline Brand et al. $(2015)^{65}$ & Long & 21 & 58.8 (SD NR) & 19 & Aneu & $5-9 \mathrm{mo}$ & Depression Skala \\
\hline Scherfler et al. $(2016)^{28}$ & Long & 14 & $46.1 \pm 12$ & 43 & Aneu (36\%) & $1 \mathrm{yr}$ & HADS \\
\hline Gerber et al. $(2016)^{42}$ & Cross & 15 & $52.7 \pm 9.8$ & 27 & Aneu & $44 \mathrm{mo}$ & $\mathrm{BDI}$ \\
\hline Taufique et al. $(2016)^{52}$ & Long & 1181 & $52.3 \pm 12.8$ & NR & NR & $1 \mathrm{yr}$ & CESD \\
\hline Kronvall et al. $(2016)^{60}$ & Long & 51 & NR & NR & Aneu & $\begin{array}{c}\text { 3-6 mo (T1) } \\
6-12 \mathrm{mo}(\mathrm{T} 2) \\
12-24 \mathrm{mo}(\mathrm{T} 3)\end{array}$ & $\begin{array}{l}\text { Psychological general well-be- } \\
\text { ing }\end{array}$ \\
\hline Pačić-Turk et al. (2016) ${ }^{64}$ & Long & 72 & $46 \pm 9.2$ & 39 & Aneu & $\begin{array}{c}11 \mathrm{mo}(\mathrm{T} 1) \\
12-48 \mathrm{mo}(\mathrm{T} 2)\end{array}$ & $\begin{array}{l}\text { Cornell personality question- } \\
\text { naire }\end{array}$ \\
\hline Colledge et al. $(2017)^{12,13}$ & Cross & 15 & $57.3 \pm 8.9$ & 27 & Aneu & $44 \mathrm{mo}$ & BDI \\
\hline Ackermark et al. $(2017)^{41}$ & Long & 93 & $50.3 \pm 11.8$ & 19.4 & Aneu & $\begin{array}{c}3 \mathrm{mo}(\mathrm{T} 1) \\
1 \mathrm{yr}(\mathrm{T} 2) \\
2-5 \mathrm{yr}(\mathrm{T} 3)\end{array}$ & BDI \\
\hline Buunk et al. $(2018)^{26}$ & Cross & 221 & $\begin{array}{c}57.0 \pm 10.0 \\
\text { (Aneu group), } \\
55.4 \pm 10.2 \\
\text { (other group) }\end{array}$ & $\begin{array}{c}31.3 \\
\text { (Aneu } \\
\text { group), } \\
58.2 \\
\text { (other } \\
\text { group) }\end{array}$ & Aneu (75\%) & $3-10 \mathrm{yr}$ & HADS \\
\hline Tölli et al. $(2018)^{25}$ & Long & 45 & $57.4 \pm 9.9$ & 22.9 & NR & $\begin{array}{c}3 \mathrm{mo}(\mathrm{T} 1) \\
6 \mathrm{mo}(\mathrm{T} 2) \\
12 \mathrm{mo}(\mathrm{T} 3)\end{array}$ & HADS \\
\hline Bründl et al. (2018) ${ }^{58}$ & Long & 21 & $\begin{array}{l}42.0-59.8 \\
\text { (mean) }\end{array}$ & $33.3-50$ & Aneu (71\%) & $\begin{array}{l}11-35 \text { day (T1) } \\
6 \text { mo (T2) }\end{array}$ & $\begin{array}{c}\text { ICD-10-Symptom-Rating Ques- } \\
\text { tionnaire }\end{array}$ \\
\hline
\end{tabular}

SD, standard deviation; SAH, subarachnoid hemorrhage; Cross, cross-sectional; NR, not reported; Aneu, aneurysmal; BDI, Beck Depression Inventory; Long, Iongitudinal; HADS, Hospital Anxiety Depression Scale; ZDS, Zung Self-rating Depression Scale; MADRS, Montgomery Åsberg Depression Rating Scale; DSMIV, Diagnostic and Statistical Manual of Mental Disorders Fourth Edition; GDS, Geriatric Depression Scale; CESD, Center for Epidemiologic Studies Depression; ICD-10, International Classification of Diseases 10th Edition.

sion was $26.3 \%$ (95\% confidence interval [Cl], $21.0 \%$ to $32.0 \%$ ). The frequency of depression in the interview and questionnaire studies were $25.0 \%$ (95\% Cl, $12.6 \%$ to $39.7 \%$ ) and 26.3\% (95\% $\mathrm{Cl}, 20.8 \%$ to $32.3 \%$ ), respectively (Figure 2). The Funnel plot did not suggest any publication bias $(P=0.434)$ (Figure 3$)$.

\section{Severity of depression after SAH}

Twelve studies ( $n=634$ ) assessed the severity of depression using the BDI. ${ }^{14-16,18-21,40,41,44,45,47}$ The weighted mean BDI value was 9.9 less than 1 year after SAH and 10.1 1-year or more after; these figures indicate mild to moderate depression. ${ }^{15}$ Nine studies $(n=1,280)$ evaluated the severity of depression using the HADS. ${ }^{27-}$ 30,33,34,36-38 The weighted mean HADS value was 5 less than 1 year after SAH and 5.4 1-year or more after; according to a previous validation study, ${ }^{66}$ both values can be interpreted as an absence of depression. ${ }^{27}$ Less commonly used mood scales were the $\mathrm{CESD}_{1}{ }^{22-24} \mathrm{MADRS}_{1}^{54,55}, \mathrm{GDS}_{1}^{56,57}$ and one study each used the ZDS $_{1}^{61}$ Depressions Skala, ${ }^{65}$ Emotional State Questionnaire, ${ }^{59}$ Cornell Personality Scale, ${ }^{64}$ and the Psychological General Well-Being Scale ${ }^{60}$ (Tables 1 and 2, Supplementary Table 2).

\section{Time course of depression after SAH}

Eight studies ( $n=539)$ assessed depression at more than one time point (Supplementary Table 3). ${ }^{18,19,23,25,29,41,44,64}$ In one study $(n=113)$, the proportion of patients with depression increased from $24.8 \%$ at discharge to $61.7 \%$ at the 12 -month follow-up. ${ }^{44}$ In another study, 72\% of patients with depressive symptoms at 3 months still had symptoms at 2 to 5 years. ${ }^{41}$ One study reported an increase in depressive symptoms from 11 months to 12 to 48 months follow-up. ${ }^{64}$ On the contrary, five studies $(n=261)$ 
Table 2. Studies of frequency and/or severity of depression after SAH

\begin{tabular}{lcccc} 
Study & Study design and setting & Timing after & Cut off \\
point & SAH & $\begin{array}{c}\text { Proportion of } \\
\text { participants } \\
\text { with depres- } \\
\text { sion (\%) }\end{array}$ & $\begin{array}{c}\text { Depressive } \\
\text { symptom } \\
\text { scores } \\
\text { (mean } \pm \text { SD) }\end{array}$ & Limitations \\
\hline
\end{tabular}

Depression measured by rating instruments/questionnaires

Alfieri et al. (2008) ${ }^{17}$

\begin{tabular}{|c|c|}
\hline Caeiro et al. $(2011)^{54}$ & $\begin{array}{l}\text { Prospective study, consecu- } \\
\text { tive admissions to an } \\
\text { academic neurosurgery } \\
\text { center }\end{array}$ \\
\hline Meyer et al. (2010) ${ }^{44}$ & Longitudinal study \\
\hline $\begin{array}{l}\text { Passier et al. (2010) })^{14}, \\
\text { Passier et al. }(2011)^{15}, \\
\text { Passier et al. }(2012)^{16}\end{array}$ & $\begin{array}{l}\text { Cross-sectional study, all } \\
\text { subjects treated with } \\
\text { clipping or coiling }\end{array}$ \\
\hline $\begin{array}{l}\text { Powell et al. (2002) })^{18} \text { and } \\
\text { Powell et al. }(2004)^{19}\end{array}$ & $\begin{array}{l}\text { Cross-sectional study, } \\
\text { consecutive admissions to } \\
\text { a neurovascular service }\end{array}$ \\
\hline Ackermark et al. $(2017)^{41}$ & $\begin{array}{l}\text { Longitudinal study, subjects } \\
\text { recruited from a clinic }\end{array}$ \\
\hline
\end{tabular}

At discharge

$12 \mathrm{mo}(\mathrm{T} 2)$

$3 \mathrm{mo}$

(T1)

$1 \mathrm{mo}(\mathrm{T} 2)$

$1 \mathrm{yr}(\mathrm{T} 3)$

$3 \mathrm{yr}(\mathrm{T} 4)$

5 yr (T5)

$\leq 4$ day

MADRS $\geq 7$

$N R$

NR

40

$3 \mathrm{mo}(\mathrm{T} 1)$

$\mathrm{BDI}>10$

$\mathrm{T} 1: 9.1$

$18 \mathrm{mo}$ (T3)

$3 \mathrm{mo}(\mathrm{T} 1)$

$1 \mathrm{yr}(\mathrm{T} 2)$

2-5 yr (T3)

Kronvall et al. (2016) $)^{60}$

Prospective study in an academic neurosurgery unit

3-6 mo (T1)

6-12 mo (T2)

$12-24 \mathrm{mo}$ (Т3)

Wong et al. (2012) ${ }^{57}$

Prospective multi-center study of consecutive admissions

Kreiter et al. (2013) $\quad$ Prospective study, consecutive admissions to an academic neurosurgery center

Boerboom et al. (2014) 22 Prospective study, consecuand Boerboom et al. tive admissions to an $(2016)^{23,24}$ academic neurosurgen center

Fontanella et al. (2003) ${ }^{50}$ Cross-sectional study, all subjects had treated anterior communicating artery bleeding aneurysm

von Vogelsang et al.

Longitudinal study,

$(2015)^{29}$ hospitalized subjects

Hellawell et al. (1999) $\quad$ Longitudinal study, subjects recruited from a neurosurgical unit

$$
3 \mathrm{mo}
$$

$3 \mathrm{mo}(\mathrm{T} 1)$

$1 \mathrm{yr}$ (T2)

$0.4 \mathrm{yr}(\mathrm{T} 1)$

$3.9 \mathrm{yr}(\mathrm{T} 2)$

$6 \mathrm{mo}$

$6 \mathrm{mo}$ (T1)

1 yr (T2)

2 yr (T3)

6 mo (T1)

1 yr (T2)

2 yr (T3)

5-9 mo subjects had treated SAH

T2: 11.4

T3: 16.3

$\mathrm{BDI} \geq 10$

T1: 39

T2: 41

T3: 54

NR

NR

NR

NR NR

CESD $\geq 16$

T1: 38

$\mathrm{T} 2: 33$

NR

T2: $9.2 \pm 6.9$

T3: $9.4 \pm 7.3$

$\mathrm{T} 1: 8.9 \pm 7.0$ Nursing home patients not included, T2: $9.3 \pm 7.1$ no data of previous mental health T3: $11.2 \pm 8.0$ problems, locus of control, optimism or social support, self-report of depressive symptoms.

T1: $15.0 \pm 3.5$ Small sample size, validity of the mood

T2: $15.3 \pm 2.9$ assessment uncertain

T3: $15.8 \pm 2.9$

7 (median) Lack of gold standard measure of depression

R Less severely affected subjects more likely to complete follow-up, subjects treated for SAH a decade ago, confounders not considered.

T2: 26.7

$T 1: 13.7 \pm 1.2$ Subjects assessed at different time $\mathrm{T} 2: 11.9 \pm 1.2$ points following $\mathrm{SAH}$.

13.8 (SD NR) Small sample size, limited generalizability to patients with other type of SAH

$H A D S \geq 8 \quad T 1: 25.0$

T2: 27.6

T3: 29.4

NR

T1: 8

T2: 9

T3: 5

NR NR

T1: $5.0 \quad$ No data on previous history of depres-

T2: 4.0 sion or use of antidepressants during

T3: 5.0 the follow-up period

(median)

NR Small sample size, high attrition rate

$1.42 \pm 0.29$ Small sample size, validity of the mood assessment uncertain, confounders not measured. 
Table 2. Continued

\begin{tabular}{|c|c|c|c|c|c|c|}
\hline Study & Study design and setting & $\begin{array}{l}\text { Timing after } \\
\text { SAH }\end{array}$ & $\begin{array}{l}\text { Cut off } \\
\text { point }\end{array}$ & $\begin{array}{l}\text { Proportion of } \\
\text { participants } \\
\text { with depres- } \\
\text { sion }(\%)\end{array}$ & $\begin{array}{l}\text { Depressive } \\
\text { symptom } \\
\text { scores } \\
\text { (mean } \pm \text { SD) }\end{array}$ & Limitations \\
\hline Pačić-Turk et al. (2016) $)^{64}$ & $\begin{array}{l}\text { Prospective study in an } \\
\text { academic neurosurgery } \\
\text { unit }\end{array}$ & $\begin{array}{c}11 \mathrm{mo}(\mathrm{T} 1) \\
12-48 \mathrm{mo}(\mathrm{T} 2)\end{array}$ & NR & NR & $\begin{array}{l}\mathrm{T} 1: 1.93 \\
\mathrm{~T} 2: 2.65\end{array}$ & $\begin{array}{l}\text { Modest sample size, validity of the } \\
\text { mood assessment uncertain }\end{array}$ \\
\hline Scherfler et al. $(2016)^{28}$ & $\begin{array}{l}\text { Longitudinal study, subjects } \\
\text { recruited from a neurologi- } \\
\text { cal intensive care unit }\end{array}$ & $1 \mathrm{yr}$ & $\mathrm{HADS}>10$ & 0 & 1 (median) & $\begin{array}{l}\text { Small sample size, selected inclusion } \\
\text { of patients without visually detect- } \\
\text { able structural lesions on MRI }\end{array}$ \\
\hline Tölli et al. $(2018)^{25}$ & $\begin{array}{l}\text { Longitudinal study, subjects } \\
\text { recruited from a neruoin- } \\
\text { tensive care unit }\end{array}$ & $1 \mathrm{yr}$ & $\mathrm{HADS} \geq 8$ & 23 & NR & Single center study, small sample size \\
\hline Orbo et al. $(2008)^{45}$ & $\begin{array}{l}\text { Longitudinal study, all } \\
\text { subjects treated with } \\
\text { clipping }\end{array}$ & $1 \mathrm{yr}$ & $\mathrm{BDI} \geq 14$ & 5 & 6 (SD NR) & Small sample size \\
\hline Preiss et al. $(2007)^{46}$ & $\begin{array}{l}\text { Longitudinal study, all } \\
\text { subjects treated with } \\
\text { coiling or clipping }\end{array}$ & $1 \mathrm{yr}$ & NR & NR & 9.4 & $\begin{array}{l}\text { Relative small sample size, potential } \\
\text { selection bias }\end{array}$ \\
\hline Haug et al. (2009) $)^{55}$ & $\begin{array}{l}\text { Prospective study, all subjects } \\
\text { treated for anterior or } \\
\text { middle cerebral artery } \\
\text { bleeding aneurysm }\end{array}$ & $1 \mathrm{yr}$ & NR & NR & 5.5 (SD NR) & $\begin{array}{l}\text { Small sample size, subjects with other } \\
\text { locations of aneurysms excluded. }\end{array}$ \\
\hline Taufique et al. $(2016)^{52}$ & $\begin{array}{l}\text { Prospective study, consecu- } \\
\text { tive admissions to an } \\
\text { academic neurosurgery } \\
\text { center }\end{array}$ & $1 \mathrm{yr}$ & CESD $\geq 16$ & 33.3 & NR & $\begin{array}{l}\text { Poor grade patients more likely to be } \\
\text { lost to follow-up. }\end{array}$ \\
\hline Mukerji et al. $(2010)^{35}$ & $\begin{array}{l}\text { Retrospective subject recruit- } \\
\text { ment, all subjects received } \\
\text { an angiogram }\end{array}$ & $\begin{array}{l}\text { Median } 13 \text { mo } \\
\text { (range, 12- } \\
\text { 266) }\end{array}$ & NR & 13 & NR & $\begin{array}{l}\text { Subjects recruited at different time } \\
\text { points following SAH, small sample } \\
\text { size }\end{array}$ \\
\hline Morris et al. (2004) ${ }^{40}$ & $\begin{array}{l}\text { Cross-sectional study, meth- } \\
\text { od and site of recruitment } \\
\text { not reported }\end{array}$ & $\begin{array}{l}\text { Average } 16 \text { mo } \\
\text { (range, } 14- \\
23 \text { ) }\end{array}$ & $\mathrm{BDI} \geq 10$ & 50 & NR & $\begin{array}{l}\text { Small sample size, opportunity sam- } \\
\text { ples, subjects recruited at different } \\
\text { time points following SAH }\end{array}$ \\
\hline Gill et al. $(2015)^{31}$ & $\begin{array}{l}\text { Cross-sectional study, } \\
\text { subjects recruited from } \\
\text { neuropsychology services, } \\
\text { charities and online } \\
\text { support network }\end{array}$ & $\begin{array}{c}\text { Average } 21.1 \\
\text { mo (range, } \\
2-58)\end{array}$ & $\mathrm{HADS} \geq 8$ & 51 & NR & $\begin{array}{l}\text { No data on those refused to partici- } \\
\text { pate, self-report data of SAH, } \\
\text { severity of injury not recorded, } \\
\text { subjects, recruited at different time } \\
\text { points following SAH. }\end{array}$ \\
\hline Fertl et al. (1999) $)^{51}$ & $\begin{array}{l}\text { Cross-sectional study, all } \\
\text { subjects treated for SAH }\end{array}$ & $\begin{array}{l}\text { Average } 22 \text { mo } \\
\quad \text { (range, NR) }\end{array}$ & $\geq 12$ & 28 & NR & $\begin{array}{l}\text { Small sample size, subjects recruited at } \\
\text { different time points following SAH }\end{array}$ \\
\hline $\begin{array}{l}\text { Kreitschmann-An- } \\
\text { dermahr et al. }(2007)^{47}\end{array}$ & $\begin{array}{l}\text { Cross-sectional study, meth- } \\
\text { od and site of recruitment } \\
\text { not reported }\end{array}$ & $\begin{array}{c}\text { Average } 27.3 \\
\text { mo (range, } \\
12-66)\end{array}$ & $\mathrm{BDI}>10$ & 37.5 & $8.33 \pm 5.85$ & $\begin{array}{l}\text { Small sample size, subjects recruited at } \\
\text { different time points following SAH }\end{array}$ \\
\hline Wong et al. (2014) ${ }^{3}$ & $\begin{array}{l}\text { Cross-sectional four centers } \\
\text { study, hospitalized subjects }\end{array}$ & $\begin{array}{l}\text { Average NR } \\
\text { (range, 1-4 yr) }\end{array}$ & - & 13 & NR & $\begin{array}{l}\text { Attrition, subjects recruited at different } \\
\text { time points following SAH, confound- } \\
\text { ers not measured, reporting bias }\end{array}$ \\
\hline $\begin{array}{l}\text { Visser-Meily et al. } \\
(2009)^{36}\end{array}$ & $\begin{array}{l}\text { Cross-sectional study, all } \\
\text { subjects had been treated } \\
\text { with coiling or clipping }\end{array}$ & $\begin{array}{l}\text { Average } 3 \text { yr } \\
\text { (range, 2-4) }\end{array}$ & $\mathrm{HADS} \geq 8$ & 23 & $4.8 \pm 3.9$ & $\begin{array}{l}\text { Selection bias as only patients still } \\
\text { alive included, subjects recruited at } \\
\text { different time points following SAH. }\end{array}$ \\
\hline Noble et al. $(2014)^{32}$ & $\begin{array}{l}\text { Cross-sectional study, } \\
\text { subjects recruited from } \\
\text { support groups }\end{array}$ & $\begin{array}{l}\text { Median } 3 \mathrm{yr} \\
\text { (range, 1-5) }\end{array}$ & $\mathrm{HADS} \geq 8$ & 45.2 & NR & $\begin{array}{l}\text { Only patients had access to internet } \\
\text { included, no data on those refused } \\
\text { to participate, lack of psychiatric } \\
\text { interviews, self-report data of SAH } \\
\text { details, subjects recruited at differ- } \\
\text { ent time points following SAH. }\end{array}$ \\
\hline Hütter et al. $(1995)^{20,21}$ & $\begin{array}{l}\text { Cross-sectional study, all } \\
\text { subjects operated for SAH }\end{array}$ & $\begin{array}{l}\text { Median } 3 \mathrm{yr} \\
\text { (range, 1-5) }\end{array}$ & $\mathrm{BDI}>10$ & 30 & NR & $\begin{array}{l}\text { Small sample size, subjects recruited at } \\
\text { different time points following SAH, ex- } \\
\text { cluded subjects had worse SAH grading. }\end{array}$ \\
\hline
\end{tabular}


Table 2. Continued

\begin{tabular}{|c|c|c|c|c|c|c|}
\hline Study & Study design and setting & $\begin{array}{l}\text { Timing after } \\
\text { SAH }\end{array}$ & $\begin{array}{l}\text { Cut off } \\
\text { point }\end{array}$ & $\begin{array}{l}\text { Proportion of } \\
\text { participants } \\
\text { with depres- } \\
\text { sion }(\%)\end{array}$ & $\begin{array}{l}\text { Depressive } \\
\text { symptom } \\
\text { scores } \\
(\text { mean } \pm S D)\end{array}$ & Limitations \\
\hline Carter et al. $(2000)^{61}$ & $\begin{array}{l}\text { Cross-sectional study, } \\
\text { consecutive admissions to } \\
\text { a tertiary medical center }\end{array}$ & $\begin{array}{l}\text { Average NR } \\
\text { (range, 1-5 yr) }\end{array}$ & $\mathrm{ZDS} \geq 50$ & 36 & 45.6 (SD NR) & $\begin{array}{l}\text { Subjects recruited at different time } \\
\text { points following SAH }\end{array}$ \\
\hline Latimer et al. $(2013)^{33}$ & $\begin{array}{l}\text { Retrospective subject } \\
\text { recruitment, all subjects } \\
\text { had anterior circulatory } \\
\text { area SAH }\end{array}$ & $40-45 \mathrm{mo}$ & NR & NR & 6.7 & $\begin{array}{l}\text { Retrospective subject recruitment, } \\
\text { selective sample, small sample size }\end{array}$ \\
\hline $\begin{array}{l}\text { Colledge et al. }(2017)^{12,13} \\
\text { and Gerber et al. } \\
(2016)^{42}\end{array}$ & $\begin{array}{l}\text { Cross-sectional study, almost } \\
\text { all subjects treated with } \\
\text { clipping }\end{array}$ & $44 \mathrm{mo}$ & NR & NR & $8.9 \pm 6.6$ & $\begin{array}{l}\text { Small sample size, subjects recruited } \\
\text { at different time points following } \\
\text { SAH, lack of psychiatric interview }\end{array}$ \\
\hline Vetkas et al. (2013) $)^{59}$ & $\begin{array}{l}\text { Retrospective study of a } \\
\text { single academic center }\end{array}$ & $\begin{array}{l}\text { Average } 4.5 \mathrm{yr} \\
\text { (range, } 1-10 \text { ) }\end{array}$ & $\mathrm{GDS} \geq 12$ & 30 & $8.4 \pm 6.9$ & $\begin{array}{l}\text { Retrospective study, subjects assessed } \\
\text { at different time points following SAH }\end{array}$ \\
\hline Buunk et al. $(2015)^{30}$ & $\begin{array}{l}\text { Cross-sectional study, all } \\
\text { subjects had been treated } \\
\text { by coiling or clipping }\end{array}$ & $\begin{array}{l}\text { Average } 4.6 \mathrm{yr} \\
\text { (range, 2-10) }\end{array}$ & $H A D S \geq 8$ & 23 & $4.2 \pm 4.3$ & $\begin{array}{l}\text { Selective sample, subjects recruited at } \\
\text { different time points following SAH }\end{array}$ \\
\hline Boerboom et al. (2017) & $\begin{array}{l}\text { Cross-sectional study, } \\
\text { hospitalized subjects }\end{array}$ & $\begin{array}{l}\text { Average } 4.7 \mathrm{yr} \\
\text { (range, NR) }\end{array}$ & $\mathrm{HADS} \geq 8$ & 15.2 & 3.5 (SD NR) & $\begin{array}{l}\text { Selection bias, small sample size, } \\
\text { subjects recruited at different time } \\
\text { points following SAH }\end{array}$ \\
\hline Salmond et al. (2006) ${ }^{48}$ & $\begin{array}{l}\text { Cross-sectional study, } \\
\text { method and site of recruit- } \\
\text { ment not reported }\end{array}$ & $\begin{array}{l}\text { Average } 68 \text { mo } \\
\text { (range, } 14- \\
99)\end{array}$ & NR & NR & $6.5 \pm 1.4$ & $\begin{array}{l}\text { Small sample size, subjects recruited at } \\
\text { different time points following SAH }\end{array}$ \\
\hline Wermer et al. (2007) & $\begin{array}{l}\text { Cross-sectional study, all } \\
\text { subjects treated with } \\
\text { clipping }\end{array}$ & $\begin{array}{c}\text { Average } 8.9 \mathrm{yr} \\
\text { (range, } 2.3- \\
18.8 \text { ) }\end{array}$ & HADS $>10$ & 9.4 & $6.2 \pm 3.1$ & $\begin{array}{l}\text { Only patients treated with clipping } \\
\text { and regained functional indepen- } \\
\text { dence included, relative young age } \\
\text { of the subjects, subjects recruited at } \\
\text { different time points following SAH. }\end{array}$ \\
\hline $\begin{array}{l}\text { von Vogelsang et al. } \\
(2013)^{34}\end{array}$ & $\begin{array}{l}\text { Retrospective subject recruit- } \\
\text { ment, subjects recruited } \\
\text { from a neurosurgical clinic }\end{array}$ & $\begin{array}{l}\text { Average } 10.1 \mathrm{yr} \\
\text { (range, } 8.8- \\
12 \text { ) }\end{array}$ & $\mathrm{HADS} \geq 8$ & 23.5 & 4.0 (median) & $\begin{array}{l}\text { Subjects recruited at different time } \\
\text { points following SAH, no data on } \\
\text { previous history of depression or use } \\
\text { of antidepressants during the follow- } \\
\text { up period }\end{array}$ \\
\hline King et al. (2009) & $\begin{array}{l}\text { Cross-sectional study, } \\
\text { subjects recruited from } \\
\text { neurosurgery clinics }\end{array}$ & NR & HADS > 10 & 9 & $4.8 \pm 3.4$ & $\begin{array}{l}\text { A single academic center study, some } \\
\text { eligible patients not participated, } \\
\text { Caucasians over-represented }\end{array}$ \\
\hline \multicolumn{7}{|l|}{$\begin{array}{l}\text { Depression measured by } \\
\text { interview }\end{array}$} \\
\hline Ljunggren et al. (1985) & $\begin{array}{l}\text { Cross-sectional study, all } \\
\text { subjects had treated SAH } \\
\text { and good neurological } \\
\text { recovery }\end{array}$ & $\begin{array}{c}\text { Average } 3.5 \mathrm{yr} \\
\text { (range, } 14 \\
\text { mo-7 yr) }\end{array}$ & - & 25 & NR & $\begin{array}{l}\text { Attrition, sampling bias, subjects } \\
\text { recruited at different time points } \\
\text { following SAH, self-report of } \\
\text { depression }\end{array}$ \\
\hline Hedlund et al. (2011) $)^{62}$ & $\begin{array}{l}\text { Prospective study, all subjects } \\
\text { had treated SAH and good } \\
\text { neurological outcome }\end{array}$ & $7 \mathrm{mo}$ & - & 21 & NR & Attrition, sampling bias \\
\hline
\end{tabular}

SAH, subarachnoid hemorrhage; SD, standard deviation; NR, not reported; MADRS, Montgomery Åsberg Depression Rating Scale; BDI, Beck Depression Inventory; CESD, Center for Epidemiologic Studies Depression; HADS, Hospital Anxiety Depression Scale; MRI, magnetic resonance imaging; ZDS, Zung Depression Scale; GDS, Geriatric Depression Scale.

found no change in depressive symptoms between the first assessment at 3, 6, or 9 months and subsequent follow-up(s) at 6, 9 months, 1, 1.5, 2, or 4 years after SAH. 18,19,23,25,29

In a cross-sectional study, the proportion of patients with depression was not statistically different from patients 2 to 5 ,
5 to 10 , and even more than 10 years after $\mathrm{SAH}$, with $8.3 \%$, $10.7 \%$, and $8.6 \%$, respectively. ${ }^{37}$ The weighted frequency of depression was 33\% up to a year after SAH and 28\% a year or more after. $^{51,53}$ There was no relationship between length of follow-up and the severity of depressive symptoms at 39 
Studies

Setting: Interview

Ljunggren et al. 1985

Random effects model

Heterogeneity: not applicable

Setting: Questionnaire

Tolli et al., 2018

Boerboom et al. 2016

Buunk et al., 2015

Gill et al. 2014

Noble \& Schenk 2014

von Vogelsang et al. 2013

Mukerii et al. 2010

Visser-Meily et al. 2009

King et al. 2009

Wermer et al. 2007

Hellawell et al. 1999

Passier et al. 2010,Passier et al. 2011,Passier et al. 2012

Orbo et al. 2008

Kreitschmann-Andermahr et al. 2007

Morris et al. 2004

Fertl et al. 1999

Hutter \& Gilsbach 1995, Hutter et al. 1995

Ackermark et al. 2017

Meyer et al. 2010

Powell et al 2002

Taufique et al. 2016

Kreiter et al. 2013

Caeiro et al. 2011

Carter et al. 2000

Wong et al. 2014

Vetkas et al. 2013

Random effects model

Heterogeneity: $I^{2}=93.901 \%, p<0.01$

Random effects model

Heterogeneity: $I^{2}=93.659 \%, p<0.01$

Estimate

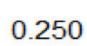

0.250

0.222

0.263

0.230

0.505

0.452

0.235

0.130

0.227

0.090

0.093

0.091

0.396

0.048

0.375

0.500

0.275

0.293

0.387

0.248

0.096

0.333

0.380

0.454

0.363

0.126

0.298

0.263

0.263
$95 \% \mathrm{Cl}$

$[0.127 ; 0.412]$

[0.126;0.397]

[0.112;0.371]

$[0.169 ; 0.377$

$[0.174 ; 0.295]$

[0 400; 0611 ]

[0.403; 0.501]

$[0.180 ; 0.297]$

$[0.064 ; 0.226]$

$[0.161 ; 0.305]$

[0.052; 0.142]

[0.072; 0.119]

$[0.025 ; 0.217]$

$[0.305 ; 0.494]$

$[0.006 ; 0.162$

$[0.227 ; 0.542]$

[0.378; 0.622]

$[0.146 ; 0.439]$

$[0.181 ; 0.427]$

$[0.288 ; 0.494]$

$[0.171 ; 0.338]$

[0.032;0.210]

$[0.306 ; 0.360]$

$[0.315 ; 0.448]$

[0.358;0.552]

[0.293;0.437]

$[0.069 ; 0.206]$

$[0.216 ; 0.391]$

$[0.208 ; 0.323]$

$[0.210 ; 0.320]$
Event Total

$\begin{array}{ll}10 & 40 \\ 10 & 40\end{array}$

40
40

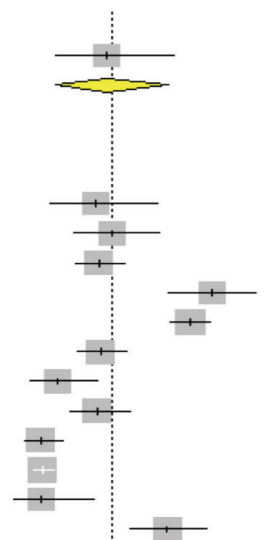

$+$
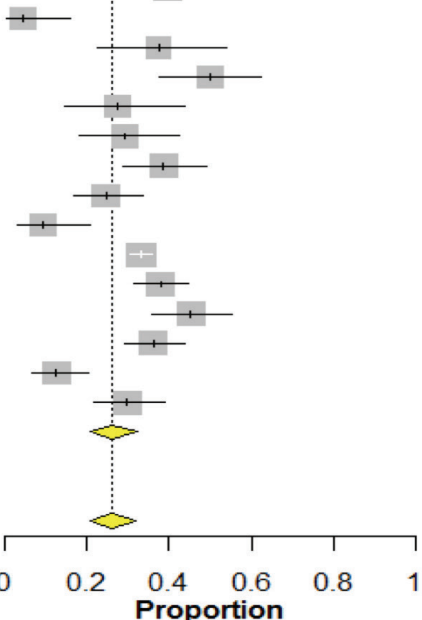

Figure 2. Meta-analysis of frequency of depression. $\mathrm{Cl}$, confidence interval.

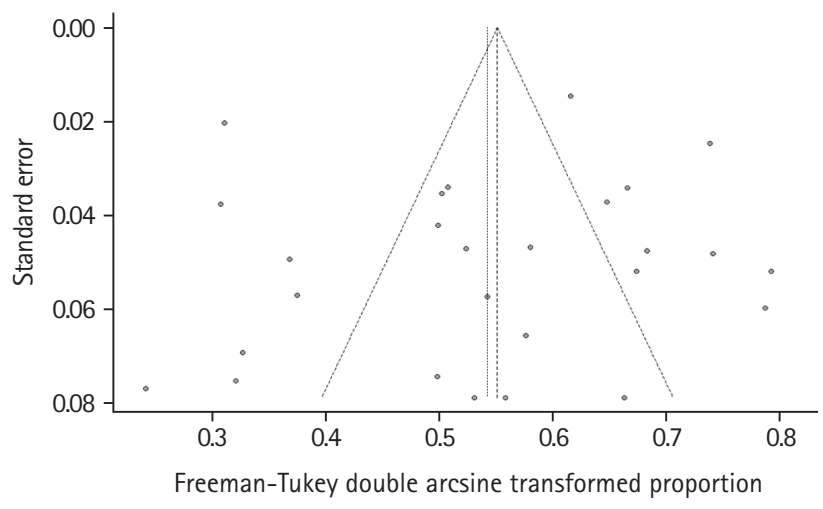

Figure 3. Funnel plot of included studies.

months after SAH in one study. ${ }^{67}$

Association between demographic factors, baseline characteristics of SAH, and depression

\section{Demographic characteristics}

Two studies explored the association between age and depression after $\mathrm{SAH}$, all with negative findings. ${ }^{20,40}$ One study reported a significant correlation between female sex and depression. ${ }^{54}$ The association between sex and depression was not significant in the other three studies $(n=314) .{ }^{34,46}$ One study found no association between depression and educational level. ${ }^{65}$ Another study reported that non-white ethnicity predicted depression (Table 3). ${ }^{53}$

\section{Premorbid conditions}

Three studies ( $n=411$ ) examined the role of history of depression prior to SAH. Associations were found between depression and previous mood disorders, ${ }^{54}$ lifetime history of major depression, or of anxiety or substance use disorder, ${ }^{62}$ and self-reported history of depression. ${ }^{53}$ One study reported that passive coping predicted post-SAH depressive symptoms (Table 3). ${ }^{41}$

Other premorbid conditions were examined in three studies. One found that depression was associated with lifetime psychiatric comorbidity. ${ }^{62}$ The second reported that non-fluency in English and nicotine use predicted depression. ${ }^{53}$ The third study observed a borderline association between depression and absence of pre-SAH dementia (Table 3). ${ }^{54}$ 
Table 3. Studies of associations between clinical features and complications of subarachnoid hemorrhage, comorbidities, biomarkers, and depression

\begin{tabular}{|c|c|c|c|}
\hline Study & Risk factors & Associations with depression & $\begin{array}{l}\text { Confounders con- } \\
\text { trolled using mul- } \\
\text { tivariate analysis }\end{array}$ \\
\hline \multicolumn{4}{|l|}{ Demographic characteristics } \\
\hline Hütter et al. (1995) ${ }^{20}$ & Age & No association & No \\
\hline Morris et al. $(2004)^{40}$ & Age & No association & No \\
\hline Caeiro et al. $(2011)^{54}$ & Sex & Female sex $(P=0.003)$ & No \\
\hline Preiss et al. $(2007)^{46}$ & Sex & No association & No \\
\hline von Vogelsang et al. $(2013)^{34}$ & Sex & No association & No \\
\hline Kreiter et al. $(2013)^{53}$ & Ethnicity & $\begin{array}{l}\text { Non-white ethnicity, }(\mathrm{OR}, 2.7 ; 95 \% \mathrm{Cl}, 1.4-5.4 ; P=0.005) \text {; non-fluency in } \\
\text { English }(\mathrm{OR}, 3.7 ; 95 \% \mathrm{Cl}, 1.7-8.2 ; P=0.001)\end{array}$ & No \\
\hline Brand et al. $(2015)^{65}$ & Education & No association & No \\
\hline \multicolumn{4}{|l|}{ Premorbid conditions } \\
\hline Caeiro et al. $(2011)^{54}$ & Psychiatric history & Past mood disorder ( $P=0.007)$, absence of pre-SAH dementia $(P=0.05)$ & No \\
\hline Kreiter et al. $(2013)^{53}$ & Psychiatric history & History of depression $(\mathrm{OR}, 3.1 ; 95 \% \mathrm{Cl}, 1.2-7.6 ; P=0.016)$ & Yes \\
\hline Hedlund et al. (2011) ${ }^{62}$ & Psychiatric history & $\begin{array}{l}\text { Lifetime affective disorder }(\mathrm{OR}, 11.9 ; 95 \% \mathrm{Cl}, 3.0-46, P=0.001) \text {, anxiety disor- } \\
\text { der }(\mathrm{OR}, 6.5 ; 95 \% \mathrm{Cl}, 1.6-26 ; P=0.008) \text {, substance use disorder }(\mathrm{OR}, 9.8 ; \\
95 \% \mathrm{Cl}, 1.5-66 ; P=0.019) \text {, or any psychiatric disorders }(\mathrm{OR}, 14.1 ; 95 \% \mathrm{Cl} \\
\text { 3.0-47; } P=0.001)\end{array}$ & Yes \\
\hline Kreiter et al. $(2013)^{53}$ & Psychiatric history & Nicotine use $(\mathrm{OR}, 2.4 ; 95 \% \mathrm{Cl}, 1.3-4.5 ; P=0.006)$ & Yes \\
\hline Ackermark et al. $(2017)^{41}$ & Premorbid personality traits & Passive coping was correlated with depressive symptoms ( $\rho=0.576, P<0.001)$. & Yes \\
\hline \multicolumn{4}{|l|}{$\begin{array}{l}\text { Clinical features and compli- } \\
\text { cations of SAH }\end{array}$} \\
\hline Hütter et al. (1995) 20,21 & Neurological outcomes & No association & No \\
\hline Morris et al. $(2004)^{40}$ & Neurological outcomes & No association & No \\
\hline Bründl et al. $(2018)^{58}$ & subtypes of SAH & $\begin{array}{l}\text { Depression symptoms were more common in aneurysmal SAH patients treat- } \\
\text { ed with microsurgury and endovascular aneurysm occlusion than those } \\
\text { with perimensencephalic } \mathrm{SAH} \text { ( } P=0.035 \text { and } P=0.016 \text { respectively). }\end{array}$ & No \\
\hline Boerboom et al. $(2014)^{22}$ & subtypes of SAH & $\begin{array}{l}\text { Aneurysmal SAH patients had a higher mean CESD score }(13.9 \pm 8.7 \text { vs. } \\
5.0 \pm 4.9, P=0.006) \text { and higher rate of depression }(44.4 \% \text { vs. } 0 \%, P=0.035) \\
\text { than perimensencephalic } \mathrm{SAH} \text {. }\end{array}$ & No \\
\hline von Vogelsang et al. $(2013)^{34}$ & Location of aneurysms & $\begin{array}{l}\text { Rupture of posterior circulation aneurysms, compared to anterior circulation } \\
\text { aneurysums, was related to a higher level of depression }(P=0.036) \text {. }\end{array}$ & No \\
\hline Hütter et al. (1995) $)^{20}$ & subtypes of SAH & No association & No \\
\hline Kreiter et al. $(2013)^{53}$ & Infarctions & SAH-related infarction predicted depression $(\mathrm{OR}, 2.1 ; 95 \% \mathrm{Cl}, 1.1-4.0 ; P=0.026)$. & Yes \\
\hline Hütter et al. (1995) $)^{21}$ & Infarctions & $\begin{array}{l}\text { Parietal and/or frontal infarcts were negatively correlated with depression } \\
\qquad(\mathrm{n}=58 ; \mathrm{F}=5.03, \mathrm{t}=2.57, P=0.03)\end{array}$ & No \\
\hline Bellebaum et al. (2004) ${ }^{49}$ & SAH treatment & $\begin{array}{l}\text { Patients treated with clips had more depressive symptoms than those treated } \\
\text { with coils }(U=73.50 ; P=0.039) \text {. }\end{array}$ & No \\
\hline Preiss et al. $(2007)^{46}$ & SAH treatment & No difference between clips and coils & No \\
\hline Fontanella et al. $(2003)^{50}$ & SAH treatment & No difference between clips and coils & No \\
\hline Latimer et al. $(2013)^{33}$ & SAH treatment & No difference between clips and coils & No \\
\hline \multicolumn{4}{|l|}{ Comorbidities } \\
\hline Boerboom et al. $(2017)^{27}$ & Cognitive function & Self-rated cognitive function $(r=0.372)$ and memory function $(r=-0.427)$ & No \\
\hline Fertl et al. (1999) $)^{51}$ & Cognitive function & Cognitive impairment $(P<0.01)$ & No \\
\hline Passier et al. (2010) $)^{14}$ & Cognitive function & depressive symptoms predicted cognitive complaints $(\beta=0.40, P<0.001)$ & Yes \\
\hline Wong et al. $(2012)^{57}$ & Cognitive function & $\begin{array}{l}\text { MoCA (Kendall's tau b coefficient } 0.191 ; P=0.027 \text { ) and MMSE (Kendall's tau } \\
\text { b coefficient } 0.198 ; P=0.024 \text { ) }\end{array}$ & No \\
\hline Brand et al. $(2015)^{65}$ & Cognitive function & No association & No \\
\hline Tölli et al. $(2018)^{25}$ & Cognitive function & No association & No \\
\hline
\end{tabular}


Table 3. Continued

\begin{tabular}{|c|c|c|c|}
\hline Study & Risk factors & Associations with depression & $\begin{array}{l}\text { Confounders con- } \\
\text { trolled using mul- } \\
\text { tivariate analysis }\end{array}$ \\
\hline Orbo et al. $(2008)^{45}$ & Cognitive function & No association & No \\
\hline Ljunggren et al. (1985) & Fatigue & Correlated with depressive symptoms $(r=0.597)$ & No \\
\hline Buunk et al. $(2018)^{26}$ & Fatigue & Correlated with depressive symptoms $(r=0.58)$ & No \\
\hline Hütter et al. (2014) ${ }^{43}$ & $\begin{array}{l}\text { Post-traumatic stress } \\
\text { disorder }\end{array}$ & $\begin{array}{l}\text { Severity of depression was correlated with scores on the IES avoidance and } \\
\text { intrusion subscales ( } r=0.45 \text { and } r=0.52 \text {, respectively). }\end{array}$ & No \\
\hline Gill et al. $(2015)^{31}$ & $\begin{array}{l}\text { Post-traumatic stress } \\
\text { disorder }\end{array}$ & $\begin{array}{l}\text { Higher rate of depression predicted greater symptoms of post-traumatic } \\
\text { stress disorder }(\beta=0.38, t=5.74, P<0.001) \text {. }\end{array}$ & Yes \\
\hline Boerboom et al. (2017) ${ }^{27}$ & Physical comorbidity & Correlated with depressive symptoms $(r=0.419)$ & No \\
\hline \multicolumn{4}{|l|}{ Functioning } \\
\hline Ackermark et al. $(2017)^{41}$ & Disability & Correlated with depressive symptoms ( $\rho=-0.343, P=0.001)$ & Yes \\
\hline Fertl et al. (1999) & Reduced working capacity & $\begin{array}{l}\text { Depression was more frequent in patients with reduced working capacity } \\
(P<0.001) \text {. }\end{array}$ & No \\
\hline \multicolumn{4}{|l|}{ Biomarkers } \\
\hline Colledge et al. $(2017)^{13}$ & Hair cortisol level & Correlated with depressive symptoms $(r=0.56)$ & No \\
\hline $\begin{array}{l}\text { Kreitschmann-Andermahr et } \\
\text { al. }(2007)^{47}\end{array}$ & Basal cortisol value & Correlated with $(r=-0.56, P<0.01)$ and predicted depression $\left(R^{2}=0.30\right)$ & Yes \\
\hline Alfieri et al. (2008) ${ }^{17}$ & APOE-ع4 & Correlated with depressive symptoms $(P<0.05)$ & No \\
\hline
\end{tabular}

OR, odds ratio; SAH, subarachnoid hemorrhage; CESD, Center for Epidemiologic Studies Depression; MoCA, Montreal Cognitive Assessment; MMSE, MiniMental State Examination; IES, Impact of Event Scale; APOE- $\varepsilon 4$, apolipoprotein E $\varepsilon 4$.

\section{Association between clinical features and complications of SAH, comorbidities, biomarkers, and depression}

\section{Clinical features and complications of SAH}

Two studies ( $n=128)$ examined the relationship between neurological outcomes and depression, with negative results (Table 3). ${ }^{20,40}$

Three studies ( $n=124)$ compared subtypes of SAH with respect to depression. Depressive symptoms were more common in patients with aneurysmal SAH treated by endovascular treatment or microsurgical clipping than in patients with perimesencephalic SAH. ${ }^{58}$ Similarly, aneurysmal SAH patients had a higher mean CESD score and higher rate of depression than their perimensencephalic counterparts in a cohort study. ${ }^{22}$ No difference in depressive symptoms between aneurysm versus other bleeding source was observed in a third study (Table 3). ${ }^{20}$

Two studies ( $n=274)$ examined the effect of infarction on the frequency of depression following SAH. SAH-related infarction predicted depression. ${ }^{53}$ Parietal and/or frontal infarcts were negatively correlated with depression (Table 3 ). ${ }^{20}$

Four studies ( $n=167)$ investigated the effect of SAH treatment. Patients treated with clips had more depressive symptoms than those treated with coils. ${ }^{49}$ The other three studies $(n=135)$ re- ported no difference in depressive symptom scores between patients who were treated by surgical clipping and by endovascular coiling. ${ }^{33,46,50}$ One study reported no significant association between measures of depression scores and time from admission until surgery. ${ }^{40}$ In a second study, rupture of posterior circulation aneurysms was related to a higher level of depression (Table 3). ${ }^{34}$

\section{Comorbidities}

Seven studies ( $n=399)$ examined the impact of cognitive function on post-SAH depression. Two studies reported an association between depressive symptoms and objective and self-rated cognitive function and memory function. ${ }^{27,57}$ Another study found depression to be more frequent in cognitively impaired patients..$^{51}$ In the fourth study, depressive symptoms were significant determinants of cognitive complaints. ${ }^{14}$ In contrast, three studies found no association between depressive symptoms and cognitive performance or impairment. ${ }^{25,45,65}$ Two studies explored the role of fatigue in post-SAH depression. Both reported a positive correlation between depressive symptoms and fatigue (Table 3). ${ }^{13,63}$

Other comorbid conditions were examined in five studies. Severity of depression was correlated with symptoms of posttraumatic stress disorder in two studies. ${ }^{31,43} \mathrm{~A}$ third study found an association between depressive symptoms and overall physical comorbidity. ${ }^{27}$ In the fourth study, disability predicted de- 
Table 4. Summary of studies reporting impact of depression after subarachnoid hemorrhage on patients' lives

\begin{tabular}{|c|c|c|c|}
\hline Study & Outcomes & Associations with outcomes & Confounders adjusted for \\
\hline \multicolumn{4}{|l|}{ Work } \\
\hline Buunk et al. $(2015)^{30}$ & Unemployment & $\begin{array}{l}\text { Unemployed patients had higher level of depression } \\
\text { (HADS-D score: } 4.98 \pm 4.57 \text { vs. } 3.01 \pm 3.41, P<0.05 \text { ). }\end{array}$ & None \\
\hline Hedlund et al. $(2011)^{62}$ & Unemployment & $\begin{array}{l}\text { Patients with a lifetime history of depression has higher } \\
\text { rate of unemployment }\left(x^{2}=5.5, P=0.019\right) \text {. }\end{array}$ & None \\
\hline Boerboom et al. $(2016)^{24}$ & Unemployment & $\begin{array}{l}\text { Depression predicted unemployment }(\mathrm{OR}, 1.126 ; 95 \% \\
\mathrm{Cl}, 1.01-1.25 ; P=0.031)\end{array}$ & Age, gender, cognitive function \\
\hline Carter et al. (2000) $)^{61}$ & Unemployment & $\begin{array}{l}\text { Depression predicted unemployment }(\mathrm{OR}, 10.5 ; 95 \% \mathrm{Cl} \\
\text { 3.3-33.7; } P<0.001)\end{array}$ & Age, physical disability, neurological deficits \\
\hline Fertl et al. (1999) $)^{51}$ & $\begin{array}{l}\text { Reduced work } \\
\text { capacity }\end{array}$ & $\begin{array}{l}\text { Depression was more common amongst patients with } \\
\text { reduced work capacity }(P<0.001) \text {. }\end{array}$ & None \\
\hline \multicolumn{4}{|c|}{ HROOL and related outcomes } \\
\hline Passier et al. (2012) $)^{16}$ & HRQOL & Depressive symptoms did not predict HROOL. & $\begin{array}{l}\text { Gender, education level, aneurysm location, } \\
\text { discharge destination, cognitive function, } \\
\text { level of impairment }\end{array}$ \\
\hline Taufique et al. $(2016)^{52}$ & HRQOL & $\begin{array}{l}\text { Depression predicted poor HRQOL }(\mathrm{OR}, 2.3 ; 95 \% \mathrm{Cl} \text {, 1.7- } \\
\text { 7.3; } P=0.02) \text {. }\end{array}$ & $\begin{array}{l}\text { Age, ethnicity, education level, history of } \\
\text { anxiety, neurological assessments, } \\
\text { dmission CT scan grading, complications }\end{array}$ \\
\hline Vetkas et al. $(2013)^{59}$ & HRQOL & $\begin{array}{l}\text { Depressive symptoms were related to lower mental } \\
\text { health component score of } \mathrm{HROOL}(\beta=-8.8, \mathrm{SE}=1.6 \text {, } \\
P<0.05) \text {. }\end{array}$ & $\begin{array}{l}\text { Anxiety, agoraphobia-panic, fatigue and } \\
\text { insomnia symptoms }\end{array}$ \\
\hline Meyer et al. (2010) $)^{44}$ & HRQOL & $\begin{array}{l}\text { Depression predicted poor } \mathrm{HROOL}(\beta=-1.80,95 \% \mathrm{Cl} \text {, } \\
-4.01 \text { to }-0.06, P=0.03) \text {. }\end{array}$ & $\begin{array}{l}\text { Gender, marital status, education, clinical } \\
\text { status on admission, functional disability }\end{array}$ \\
\hline King et al. (2009) $)^{37}$ & HRQOL & $\begin{array}{l}\text { Depressive symptoms was correlated with lower } \mathrm{HROOL} \\
(\rho=-0.52, P<0.001) \text {. }\end{array}$ & Disability, anxiety symptoms \\
\hline Brand et al. $(2015)^{65}$ & HROOL & No association & None \\
\hline Fertl et al. (1999) $)^{51}$ & Satisfaction in life & $\begin{array}{l}\text { Depressive symptoms was correlated with lower } \\
\text { satisfaction in life }(r=-0.46, P<0.01) \text {. }\end{array}$ & None \\
\hline \multicolumn{4}{|l|}{ Functional outcomes } \\
\hline Wong et al. $(2013)^{56}$ & $\begin{array}{l}\text { Functional } \\
\text { outcomes }\end{array}$ & $\begin{array}{l}\text { Depression predicted unfavorable outcome }(\mathrm{OR}, 1.24 \text {; } \\
95 \% \mathrm{Cl}, 1.1-1.3 ; P<0.001) \text {. }\end{array}$ & Cognitive deficits, neurological deficits \\
\hline Buunk et al. $(2018)^{26}$ & $\begin{array}{l}\text { Functional } \\
\text { outcomes }\end{array}$ & No association & Fatigue and anxiety symptoms \\
\hline Hütter et al. $(1995)^{20}$ & $\begin{array}{l}\text { Functional impair- } \\
\text { ment in daily life }\end{array}$ & $\begin{array}{l}\text { Depression was correlated with functional impairment } \\
\text { in daily life }(r=0.63, P<0.001) \text {. }\end{array}$ & None \\
\hline \multicolumn{4}{|l|}{ Other outcomes } \\
\hline Buunk et al. $(2015)^{30}$ & $\begin{array}{l}\text { Leisure and social } \\
\text { activities }\end{array}$ & $\begin{array}{l}\text { HADS-D score correlated with problems in leisure } \\
(\mathrm{r}=0.45, P<0.01) \text { and social }(\mathrm{r}=0.51, P<0.01) \text { activities. }\end{array}$ & None \\
\hline Carter et al. $(2000)^{61}$ & $\begin{array}{l}\text { Reintegration to } \\
\text { normal living }\end{array}$ & $\begin{array}{l}\text { Depression predicted reintegration to normal living (OR, } \\
15.2 ; 95 \% \mathrm{Cl}, 6.4-36.2 ; P<0.001) \text {. }\end{array}$ & Age, physical disability, neurological deficits \\
\hline Passier et al. (2011) $)^{15}$ & Fatigue & $\begin{array}{l}\text { Depressive symptoms predicted severity of fatigue } \\
(\mathrm{F}=4.10, P=0.046) \text {. }\end{array}$ & Level of impairment \\
\hline
\end{tabular}

HADS-D, Hospital Anxiety Depression Scale-depression subscale; OR, odd ratio; HRQOL, health-related quality of life; CT, computed tomography; SE, standard error.

pressive symptoms. ${ }^{41}$ Depression was more frequent in patients with reduced working capacity in the fifth study (Table 3). ${ }^{51}$

\section{Biological markers}

Three studies ( $n=93$ ) assessed the relationship between biological markers and depression following SAH. One reported an association between hair cortisol level and depressive symptoms. ${ }^{13}$ Another found that depression was correlated with basal cortisol value, which also predicted depression. ${ }^{47}$ The third study found a positive correlation between apolipoprotein E $\varepsilon 4$ (APOE- $\varepsilon 4$ ) levels and depressive symptoms (Table 3). ${ }^{17}$ 
Impact of depression after SAH on patients' lives Five studies ( $n=685$ ) examined the impact of depression on work-related issues. Two studies reported that depression or depressive symptoms were more frequent in patients who were unemployed or with reduced working capacity. ${ }^{30,51}$ Lifetime history of major depression and/or post-traumatic stress disorder reduced the likelihood of returning to gainful employment. ${ }^{62}$ Two studies concluded that depression or depressive symptoms predicted unemployment (Table 4). ${ }^{24,61}$

Seven studies $(n=1,642)$ looked at health-related quality of life (HROOL) or life satisfaction. Two studies found a negative association between depressive symptoms and HRQOL or satisfaction with life. ${ }^{16,51}$ Four studies found that depression and depressive symptoms predicted poor overall HROOL or the mental health component of HROOL. ${ }^{37,44,52,59}$ One small-scale study reported no association between depression scores and quality of life (Table 4). ${ }^{65}$

Three studies ( $n=399)$ explored how depression influenced functional outcomes. Depression was correlated with self-rated functional impairment in daily life and with the impact of these impairments. ${ }^{20}$ Depression predicted poor functional outcomes a year after $\mathrm{SAH}_{1}^{56}$ but this was not confirmed 3 to 10 years after SAH (Table 4). ${ }^{26}$

Other outcomes were assessed in three studies $(n=489)$. Associations were found between depression and problems with leisure and social activities, ${ }^{30}$ failure to resume previous level of daily life, ${ }^{61}$ and fatigue (Table 4). ${ }^{15}$

\section{Discussion}

To the best of our knowledge, this was the first systematic review of depression after SAH. The weighted frequency of depression following SAH was $28.1 \%$. The severity of depressive symptoms was mild to moderate. Depression after SAH seems to run a chronic course and its frequency does not decrease with time. A host of demographic variables, premorbid and comorbid conditions, as well as clinical features and complications of SAH are related to the risk of depression. Depression has negative impacts on the daily lives of patients with SAH.

The weighted frequency of depression of $28 \%$ is similar to the frequency of depression after stroke in general (31\%)..$^{68}$ The frequency of depression in the included studies varied greatly, probably due to differences in the methods of assessment, patients' characteristics, and timing of the assessment. The variation in results was more prominent in studies that used questionnaires (0\% to $62 \%)$ than in those that used interviews to detect depression (20\% to 25\%) (Tables 2 and 4).

Interviews give a clinical diagnosis whereas questionnaires will assess depressive symptomatology rather than clinical depression. Also, the number of interview studies was considerably smaller.

Longitudinal studies confirmed that depression and depressive symptoms in the later stages of SAH were at least as frequent and severe as in the early stage. Depressive symptoms persisted for long periods after SAH in $72 \%$ of patients. ${ }^{41}$ Depression after stroke in general is also a chronic condition, with a prevalence and incidence of around $30 \%$ and $15 \%$ at 1 to 15 years post-stroke. ${ }^{69}$

The development of depression after SAH is associated with a variety of factors. The studies included in this review found relationships between depression and female sex, premorbid depression, anxiety, substance use, any psychiatric disorder, and coping styles. Comorbid cognitive impairment, fatigue, post-traumatic stress disorder, and physical disability also increased the risk of depression. The role of the features and complications of SAH in the development of depression was rarely explored, one study suggested that aneurysmal SAH and infarction may be related to depression. The findings on the impact of neurological deficits and treatment modalities for aneurysm repair on depression were inconclusive. Most of the above risk factors have also been found to be related to depression in stroke in general..$^{70}$ Further research on psychosocial factors such as pre-stroke life events and the quality of family and social support are warranted. ${ }^{70}$

Pituitary dysfunction may occur in up to one in three patients after $\mathrm{SAH}^{71}$ and could contribute to the development of depression. $^{72}$ In support of this theory, an association between low basal cortisol levels and depression has been reported in patients after SAH. ${ }^{47}$ One small-scale study reported a possible link between the APOE- $\varepsilon 4$ allele and depression. ${ }^{17}$ Interestingly, hypercortisolemia, blunted cortisol awakening response, ${ }^{73}$ and APOE polymorphisms ${ }^{74}$ increase the risk of post-stroke depression.

Post-SAH depression was significantly related to functional impairment, unemployment ${ }^{75}$ or reduced working capacity, and poor HRQOL. Data on the impact of depression on the costs of hospitalization and mortality related to SAH are lacking. ${ }^{70}$

In terms of treatment of post-SAH depression, only one small-scale, open label trial of mindfulness-based psychotherapy has been published. Proper randomized control trials with antidepressants and other treatment modalities are clearly needed. Antidepressants seem to be commonly prescribed in patents with $\mathrm{SAH}$, in a population-based cohort of 940 patients with SAH, 27\% had continuous antidepressant use. ${ }^{76}$ On the other hand, the use of selective serotonin reuptake inhibitors, a commonly prescribed antidepressant, in general population was associated with increased risk of intracranial hemorrhage, particularly in the first 30 days of use and when used currently 
with anticoagulants..$^{77}$ Antidepressants are effective in the treatment ${ }^{78}$ and prevention of post-stroke depression, ${ }^{79}$ and non-pharmacological treatment modalities including ecosystem-focused therapy, life review therapy, problem solving therapy, meridian acupressure, transcranial magnetic stimulation, music therapy, exercise, light therapy, motivational interviewing, and robotic-assisted neurorehabilitation could also be trialed. ${ }^{80}$

This systematic review has several methodological strengths. An extensive search strategy was used so it is unlikely that relevant studies were missed. Two authors extracted pre-specified data independently, thus reducing the chance that any errors in data extraction would have gone undetected. A major limitation is the inclusion only of studies published in English.

There were several methodological shortcomings in the included studies that weakened the robustness of the review. First, the study design was heterogeneous, including longitudinal, ${ }_{1}^{27}$ cross-sectional, ${ }_{1}^{26}$ or retrospective ${ }^{32}$ single site cohorts. Second, while most studies recruited hospitalized ${ }^{26}$ subjects, some employed clinic, ${ }_{1}^{33,38,42}$ population, ${ }_{1}^{60}$ or support group ${ }^{30,31}$ based sampling. Third, a number of studies involved bias sample, such as particular location, ${ }^{32,54,81}$ investigation ${ }^{3}$ or treatment received, ${ }^{11,37,45}$ or neurological outcome ${ }^{65,82}$ of SAH. Fourth, many studies had small sample size. Fifth, the timing of mood assessment various varied from acute ${ }^{53,55}$ to chronic stage ${ }^{33}$ of SAH recovery. In addition, most of them assessed depression only once; in cross-sectional studies, subjects were assessed at different time point following SAH. Sixth, most studies used scales to measure depression, whereas some employed, a single question, $^{83}$ or clinical interview. ${ }^{61,62}$ Seventh, some authors described the baseline characteristics of SAH but did not relate them to the presence or severity of depression. Eighth, the majority of studies did not measure and adjust for potential confounders, such as personality, level of social support, recent life events, or previous depression with multivariate analysis. Future studies should consider prospective multi-center design, careful and non-selective sampling method, large sample size, assessment of depression at multiple time points with structural psychiatric interview, and detailed measurement and analysis of demographic and clinical characteristics and other possible confounding factors.

\section{Implication for clinicians}

Clinicians involved in the long-term care of SAH survivors need to be aware that post-SAH depression is common, runs a chronic course, and has a negative impact on patients' lives. Clinicians should routinely ask about depression when they review SAH patients and they should refer patients with suspected depression for psychological and/or psychiatric evaluation and treatment.

\section{Directions for future research}

More longitudinal studies are needed to determine the time course of depression after SAH using standardized diagnostic interviews. More studies assessing the relationship between depression and demographics, premorbid and comorbid conditions and complications of SAH are required to elucidate the relationship between depression and the consequences of SAH. Research on the association between depression and pituitary dysfunction, changes in neurotransmitter metabolism, and disruption of brain circuits after SAH is also warranted to clarify the pathogenesis of post-SAH depression. Finally, randomized controlled clinical trials on potential treatments for SAH-related depression are also needed.

\section{Conclusions}

Depression is common after SAH and seems to persist. The development of depression after SAH is associated with a variety of factors. Post-SAH depression had negative impacts on patients' daily life. Further research is needed to clarify its time course and identify the neuroendocrine and neurochemical factors and brain circuits associated with the development of post-SAH depression. Randomized controlled treatment trials targeting SAH-related depression are warranted.

\section{Supplementary materials}

Supplementary materials related to this article can be found online at https://doi.org/10.5853/jos.2019.02103.

\section{Disclosure}

The authors have no financial conflicts of interest.

\section{References}

1. Macdonald RL, Schweizer TA. Spontaneous subarachnoid haemorrhage. Lancet 2017;389:655-666.

2. Al-Khindi T, Macdonald RL, Schweizer TA. Cognitive and functional outcome after aneurysmal subarachnoid hemorrhage. Stroke 2010;41:e519-e536.

3. Wong GK, Lam SW, Chan SS, Lai M, Tse PP, Mok V, et al. Neuropsychiatric disturbance after aneurysmal subarachnoid hemorrhage. J Clin Neurosci 2014;21:1695-1698.

4. Rickards H. Depression in neurological disorders: an update. Curr Opin Psychiatry 2006;19:294-298. 
5. Fann JR, Hart T, Schomer KG. Treatment for depression after traumatic brain injury: a systematic review. J Neurotrauma 2009;26:2383-2402.

6. Robinson RG, Jorge RE. Post-stroke depression: a review. Am J Psychiatry 2016;173:221-231.

7. Kutlubaev MA, Barugh AJ, Mead GE. Fatigue after subarachnoid haemorrhage: a systematic review. J Psychosom Res 2012;72:305-310.

8. Von Elm E, Altman DG, Egger M, Pocock SJ, Gøtzsche PC, Vandenbroucke JP, et al. The Strengthening the Reporting of Observational Studies in Epidemiology (STROBE) statement: guidelines for reporting observational studies. J Clin Epidemiol 2008;61:344-349.

9. Freeman MF, Tukey JW. Transformations related to the angular and the square root. Ann Math Stat 1950;21:607-611.

10. IntHout J, loannidis JP, Borm GF. The Hartung-Knapp-SidikJonkman method for random effects meta-analysis is straightforward and considerably outperforms the standard DerSimonian-Laird method. BMC Med Res Methodol 2014; $14: 25$.

11. Fleiss JL. Analysis of data from multiclinic trials. Control Clin Trials 1986;7:267-275.

12. Colledge $F$, Brand $S$, Pühse $U$, Holsboer-Trachsler E, Zimmerer $\mathrm{S}$, Schleith $\mathrm{R}$, et al. A twelve-week moderate exercise programme improved symptoms of depression, insomnia, and verbal learning in post-aneurysmal subarachnoid haemorrhage patients: a comparison with meningioma patients and healthy controls. Neuropsychobiology 2017;76:59-71.

13. Colledge F, Brand S, Zimmerer S, Pühse U, Holsboer-Trachsler E, Gerber M. In individuals following aneurysmal subarachnoid haemorrhage, hair cortisol concentrations are higher and more strongly associated with psychological functioning and sleep complaints than in healthy controls. Neuropsychobiology 2017;75:12-20.

14. Passier PE, Visser-Meily JM, van Zandvoort MJ, Post MW, Rinkel GJ, van Heugten C. Prevalence and determinants of cognitive complaints after aneurysmal subarachnoid hemorrhage. Cerebrovasc Dis 2010;29:557-563.

15. Passier PE, Post MW, van Zandvoort MJ, Rinkel GJ, Lindeman $E_{1}$ Visser-Meily JM. Predicting fatigue 1 year after aneurysmal subarachnoid hemorrhage. J Neurol 2011;258:1091-1097.

16. Passier PE, Visser-Meily JM, van Zandvoort MJ, Rinkel GJ, Lindeman E, Post MW. Predictors of long-term health-related quality of life in patients with aneurysmal subarachnoid hemorrhage. NeuroRehabilitation 2012;30:137-145.

17. Alfieri $A$, Unterhuber $V$, Pircher $M$, Schwarz $A$, Gazzeri R, Reinert $M$, et al. Psychosocial and neurocognitive performance after spontaneous nonaneurysmal subarachnoid hemorrhage re- lated to the APOE-epsilon4 genotype: a prospective 5-year follow-up study. J Neurosurg 2008;109:1019-1026.

18. Powell J, Kitchen N, Heslin J, Greenwood R. Psychosocial outcomes at three and nine months after good neurological recovery from aneurysmal subarachnoid haemorrhage: predictors and prognosis. J Neurol Neurosurg Psychiatry 2002;72: 772-781.

19. Powell J, Kitchen N, Heslin J, Greenwood R. Psychosocial outcomes at 18 months after good neurological recovery from aneurysmal subarachnoid haemorrhage. J Neurol Neurosurg Psychiatry 2004;75:1119-1124.

20. Hütter BO, Gilsbach JM. Introspective capacities in patients with cognitive deficits after subarachnoid hemorrhage. J Clin Exp Neuropsychol 1995;17:499-517.

21. Hütter BO, Gilsbach JM, Kreitschmann I. Quality of life and cognitive deficits after subarachnoid haemorrhage. $\mathrm{Br} J \mathrm{Neu}-$ rosurg 1995;9:465-475.

22. Boerboom $W$, Heijenbrok-Kal MH, Khajeh $L$, van Kooten $F$, Ribbers GM. Differences in cognitive and emotional outcomes between patients with perimesencephalic and aneurysmal subarachnoid haemorrhage. J Rehabil Med 2014;46: 28-32.

23. Boerboom W, Heijenbrok-Kal MH, Khajeh $L$, van Kooten $F$, Ribbers GM. Long-term functioning of patients with aneurysmal subarachnoid hemorrhage: a 4-yr follow-up study. Am J Phys Med Rehabil 2016;95:112-120.

24. Boerboom W, Heijenbrok-Kal MH, van Kooten $F$, Khajeh $L$, Ribbers GM. Unmet needs, community integration and employment status four years after subarachnoid haemorrhage. J Rehabil Med 2016;48:529-534.

25. Tölli A, Höybye $C$, Bellander BM, Johansson F, Borg J. The effect of time on cognitive impairments after non-traumatic subarachnoid haemorrhage and after traumatic brain injury. Brain Inj 2018;32:1465-1476.

26. Buunk AM, Groen RJM, Wijbenga RA, Ziengs $A L$, Metzemaekers JDM, van Dijk JMC, et al. Mental versus physical fatigue after subarachnoid hemorrhage: differential associations with outcome. Eur J Neurol 2018;25:1313-1319.e113.

27. Boerboom W, van Zandvoort MJ, van Kooten F, Khajeh $L$, Visser-Meily JM, Ribbers GM, et al. Long-term fatigue after perimesencephalic subarachnoid haemorrhage in relation to cognitive functioning, mood and comorbidity. Disabil Rehabil 2017;39:928-933.

28. Scherfler C, Schiefecker AJ, Delazer M, Beer R, Bodner T, Spinka $\mathrm{G}$, et al. Longitudinal profile of iron accumulation in good-grade subarachnoid hemorrhage. Ann Clin Transl Neurol 2016;3:781-790.

29. von Vogelsang AC, Forsberg $C$, Svensson M, Wengström Y. 
Patients experience high levels of anxiety 2 years following aneurysmal subarachnoid hemorrhage. World Neurosurg 2015;83:1090-1097.

30. Buunk AM, Groen RJ, Veenstra WS, Spikman JM. Leisure and social participation in patients 4-10 years after aneurysmal subarachnoid haemorrhage. Brain Inj 2015;29:1589-1596.

31. Gill IJ, Mullin S, Simpson J. Are metacognitive processes associated with posttraumatic stress symptom severity following acquired brain injury? Disabil Rehabil 2015;37:692-700.

32. Noble AJ, Schenk T. Psychological distress after subarachnoid hemorrhage: patient support groups can help us better detect it. J Neurol Sci 2014;343:125-131.

33. Latimer SF, Wilson FC, McCusker CG, Caldwell SB, Rennie I. Subarachnoid haemorrhage (SAH): long-term cognitive outcome in patients treated with surgical clipping or endovascular coiling. Disabil Rehabil 2013;35:845-850.

34. von Vogelsang $A C$, Svensson $M$, Wengström $Y$, Forsberg $C$. Cognitive, physical, and psychological status after intracranial aneurysm rupture: a cross-sectional study of a Stockholm case series 1996 to 1999. World Neurosurg 2013;79: 130-135.

35. Mukerji N, Holliman D, Baisch S, Noble A, Schenk T, Nath F. Neuropsychologic impact of treatment modalities in subarachnoid hemorrhage: clipping is no different from coiling. World Neurosurg 2010;74:129-138.

36. Visser-Meily JM, Rhebergen ML, Rinkel GJ, van Zandvoort MJ, Post MW. Long-term health-related quality of life after aneurysmal subarachnoid hemorrhage: relationship with psychological symptoms and personality characteristics. Stroke 2009;40:1526-1529.

37. King JT Jr, Tsevat J, Roberts MS. Measuring preference-based quality of life using the EuroOol EQ-5D in patients with cerebral aneurysms. Neurosurgery 2009;65:565-573.

38. Wermer MJ, Kool H, Albrecht KW, Rinkel GJ; Aneurysm Screening after Treatment for Ruptured Aneurysms Study Group. Subarachnoid hemorrhage treated with clipping: long-term effects on employment, relationships, personality, and mood. Neurosurgery 2007;60:91-98.

39. Hellawell DJ, Taylor R, Pentland B. Persisting symptoms and carers' views of outcome after subarachnoid haemorrhage. Clin Rehabil 1999;13:333-340.

40. Morris PG, Wilson JT, Dunn L. Anxiety and depression after spontaneous subarachnoid hemorrhage. Neurosurgery 2004; 54:47-54.

41. Ackermark PY, Schepers VP, Post MW, Rinkel GJ, Passier PE, Visser-Meily JM. Longitudinal course of depressive symptoms and anxiety after aneurysmal subarachnoid hemorrhage. Eur J Phys Rehabil Med 2017;53:98-104.
42. Gerber M, Colledge F, Pühse U, Holsboer-Trachsler E, Zimmerer S, Brand S. Sleep quality, sleep EEG pattern, mental well-being and cortisol secretion in patients with ruptured aneurysm post-treatment: a comparison with post-surgery meningioma patients and controls. Neuropsychobiology 2016;73:148-159.

43. Hütter BO, Kreitschmann-Andermahr I. Subarachnoid hemorrhage as a psychological trauma. J Neurosurg 2014;120: 923-930.

44. Meyer B, Ringel F, Winter Y, Spottke A, Gharevi N, Dams J, et al. Health-related quality of life in patients with subarachnoid haemorrhage. Cerebrovasc Dis 2010;30:423-431.

45. Orbo M, Waterloo K, Egge A, Isaksen J, Ingebrigtsen T, Romner $B$. Predictors for cognitive impairment one year after surgery for aneurysmal subarachnoid hemorrhage. J Neurol 2008;255:1770-1776.

46. Preiss M, Koblihova J, Netuka D, Klose J, Charvat F, Benes V. Ruptured cerebral aneurysm patients treated by clipping or coiling: comparison of long-term neuropsychological and personality outcomes. Zentralb/ Neurochir 2007;68:169-175.

47. Kreitschmann-Andermahr I, Poll E, Hutter BO, Reineke A, Kristes $S$, Gilsbach JM, et al. Quality of life and psychiatric sequelae following aneurysmal subarachnoid haemorrhage: does neuroendocrine dysfunction play a role? Clin Endocrinol (Oxf) 2007;66:833-837.

48. Salmond $\mathrm{CH}$, DeVito $\mathrm{EE}$, Clark L, Menon DK, Chatfield DA, Pickard JD, et al. Impulsivity, reward sensitivity, and decisionmaking in subarachnoid hemorrhage survivors. J Int Neuropsychol Soc 2006;12:697-706.

49. Bellebaum C, Schäfers L, Schoch B, Wanke I, Stolke D, Forsting $M$, et al. Clipping versus coiling: neuropsychological follow up after aneurysmal subarachnoid haemorrhage (SAH). J Clin Exp Neuropsychol 2004;26:1081-1092.

50. Fontanella $M$, Perozzo $P$, Ursone $R$, Garbossa D, Bergui $M$. Neuropsychological assessment after microsurgical clipping or endovascular treatment for anterior communicating artery aneurysm. Acta Neurochir (Wien) 2003;145:867-872.

51. Fertl $E_{1}$ Killer M, Eder H, Linzmayer L, Richling B, Auff E. Long-term functional effects of aneurysmal subarachnoid haemorrhage with special emphasis on the patient's view. Acta Neurochir (Wien) 1999;141:571-577.

52. Taufique Z, May T, Meyers E, Falo C, Mayer SA, Agarwal S, et al. Predictors of poor quality of life 1 year after subarachnoid hemorrhage. Neurosurgery 2016;78:256-264.

53. Kreiter KT, Rosengart AJ, Claassen J, Fitzsimmons BF, Peery $\mathrm{S}$, $\mathrm{Du} Y \mathrm{Y}_{\text {, et }}$ al. Depressed mood and quality of life after subarachnoid hemorrhage. J Neurol Sci 2013;335:64-71.

54. Caeiro L, Santos CO, Ferro JM, Figueira ML. Neuropsychiatric disturbances in acute subarachnoid haemorrhage. Eur J Neu- 
rol 2011;18:857-864.

55. Haug $T$, Sorteberg A, Sorteberg W, Lindegaard KF, Lundar T, Finset $A$. Cognitive functioning and health related quality of life after rupture of an aneurysm on the anterior communicating artery versus middle cerebral artery. $\mathrm{Br} J$ Neurosurg 2009;23:507-515.

56. Wong GK, Lam SW, Ngai K, Wong A, Siu D, Poon WS, et al. Cognitive domain deficits in patients with aneurysmal subarachnoid haemorrhage at 1 year. J Neurol Neurosurg Psychiatry 2013;84:1054-1058.

57. Wong GK, Lam S, Ngai K, Wong A, Mok V, Poon WS, et al. Evaluation of cognitive impairment by the Montreal cognitive assessment in patients with aneurysmal subarachnoid haemorrhage: prevalence, risk factors and correlations with 3 month outcomes. J Neurol Neurosurg Psychiatry 2012;83:1112-1117.

58. Bründl E, Schödel P, Bele S, Proescholdt M, Scheitzach J, Zeman $F$, et al. Treatment of spontaneous subarachnoid hemorrhage and self-reported neuropsychological performance at 6 months: results of a prospective clinical pilot study on good-grade patients. Turk Neurosurg 2018;28:369-388.

59. Vetkas A, Lepik T, Eilat T, Rätsep T, Asser T. Emotional health and quality of life after aneurysmal subarachnoid hemorrhage. Acta Neurochir (Wien) 2013;155:1107-1114.

60. Kronvall E, Sonesson B, Valdemarsson S, Siemund R, Säveland $\mathrm{H}$, Nilsson $0 \mathrm{G}$. Reduced quality of life in patients with pituitary dysfunction after aneurysmal subarachnoid hemorrhage: a prospective longitudinal study. World Neurosurg 2016;88:83-91.

61. Carter BS, Buckley D, Ferraro R, Rordorf G, Ogilvy CS. Factors associated with reintegration to normal living after subarachnoid hemorrhage. Neurosurgery 2000;46:1326-1334.

62. Hedlund $M$, Zetterling $M$, Ronne-Engström $E_{1}$ Carlsson $M$, Ekselius L. Depression and post-traumatic stress disorder after aneurysmal subarachnoid haemorrhage in relation to lifetime psychiatric morbidity. Br J Neurosurg 2011;25:693-700.

63. Ljunggren $B$, Sonesson $B$, Säveland $H$, Brandt L. Cognitive impairment and adjustment in patients without neurological deficits after aneurysmal SAH and early operation. J Neurosurg 1985;62:673-679.

64. Pačić-Turk L, Šulentić T, Havelka Meštrović A, Paladino J, Mrak G. Personality changes following brain artery aneurysm surgery. Acta Clin Croat 2016;55:565-578.

65. Brand S, Zimmerer S, Kalak N, Planta SV, Schwenzer-Zimmerer K, Müller AA, et al. Compared to controls, patients with ruptured aneurysm and surgical intervention show increase in symptoms of depression and lower cognitive performance, but their objective sleep is not affected. World $J$ Biol Psychiatry 2015;16:96-105.
66. Spinhoven $P$, Ormel J, Sloekers PP, Kempen Gl, Speckens AE, Van Hemert AM. A validation study of the Hospital Anxiety and Depression Scale (HADS) in different groups of Dutch subjects. Psychol Med 1997;27:363-370.

67. Madureira S, Canhão $P$, Guerreiro M, Ferro JM. Cognitive and emotional consequences of perimesencephalic subarachnoid hemorrhage. J Neurol 2000;247:862-867.

68. Hackett ML, Pickles K. Part I: frequency of depression after stroke: an updated systematic review and meta-analysis of observational studies. Int J Stroke 2014;9:1017-1025.

69. Ayerbe $L$, Ayis S, Crichton S, Wolfe CD, Rudd AG. The natural history of depression up to 15 years after stroke: the South London Stroke Register. Stroke 2013;44:1105-1110.

70. Villa RF, Ferrari F, Moretti A. Post-stroke depression: mechanisms and pharmacological treatment. Pharmacol Ther 2018; 184:131-144.

71. Vespa P; Participants in the International Multi-Disciplinary Consensus Conference on the Critical Care Management of Subarachnoid Hemorrhage. Endocrine function following acute SAH. Neurocrit Care 2011;15:361-364.

72. Plotsky PM, Owens MJ, Nemeroff CB. Psychoneuroendocrinology of depression: hypothalamic-pituitary-adrenal axis. Psychiatr Clin North Am 1998;21:293-307.

73. Levada OA, Troyan AS. Poststroke depression biomarkers: a narrative review. Front Neurol 2018;9:577.

74. Li XB, Wang J, Xu AD, Huang JM, Meng LQ, Huang RY, et al. Apolipoprotein $\mathrm{E}$ polymorphisms increase the risk of poststroke depression. Neural Regen Res 2016;11:1790-1796.

75. Al Yassin A, Ouyang B, Temes R. Depression and anxiety following aneurysmal subarachnoid hemorrhage are associated with higher six-month unemployment rates. J Neuropsychiatry Clin Neurosci 2017;29:67-69.

76. Huttunen J, Lindgren $A$, Kurki MI, Huttunen $T$, Frösen J, von Und $\mathrm{Zu}$ Fraunberg $M$, et al. Antidepressant use after aneurysmal subarachnoid hemorrhage: a population-based casecontrol study. Stroke 2016;47:2242-2248.

77. Renoux C, Vahey S, Dell'Aniello S, Boivin JF. Association of selective serotonin reuptake inhibitors with the risk for spontaneous intracranial hemorrhage. JAMA Neurol 2017;74:173-180.

78. Yi ZM, Liu F, Zhai SD. Fluoxetine for the prophylaxis of poststroke depression in patients with stroke: a meta-analysis. Int J Clin Pract 2010;64:1310-1317.

79. Mead GE, Hsieh CF, Lee R, Kutlubaev M, Claxton A, Hankey GJ, et al. Selective serotonin reuptake inhibitors for stroke recovery: a systematic review and meta-analysis. Stroke 2013;44:844-850.

80. Hadidi NN, Huna Wagner RL, Lindquist R. Nonpharmacological treatments for post-stroke depression: an integrative re- 
view of the literature. Res Gerontol Nurs 2017;10:182-195.

81. Joo JH, Morales KH, de Vries HF, Gallo JJ. Disparity in use of psychotherapy offered in primary care between older African-American and white adults: results from a practicebased depression intervention trial. J Am Geriatr Soc 2010; 58:154-160.

82. Hedlund $M$, Zetterling $M$, Ronne-Engstrom $E$, Ekselius $L$, Carlsson M. Perceived recovery after aneurysmal subarach- noid haemorrhage in individuals with or without depression. J Clin Nurs 2010;19:1578-1587.

83. Krajewski K, Dombek S, Martens T, Köppen J, Westphal M, Regelsberger J. Neuropsychological assessments in patients with aneurysmal subarachnoid hemorrhage, perimesencephalic SAH, and incidental aneurysms. Neurosurg Rev 2014; 37:55-62. 


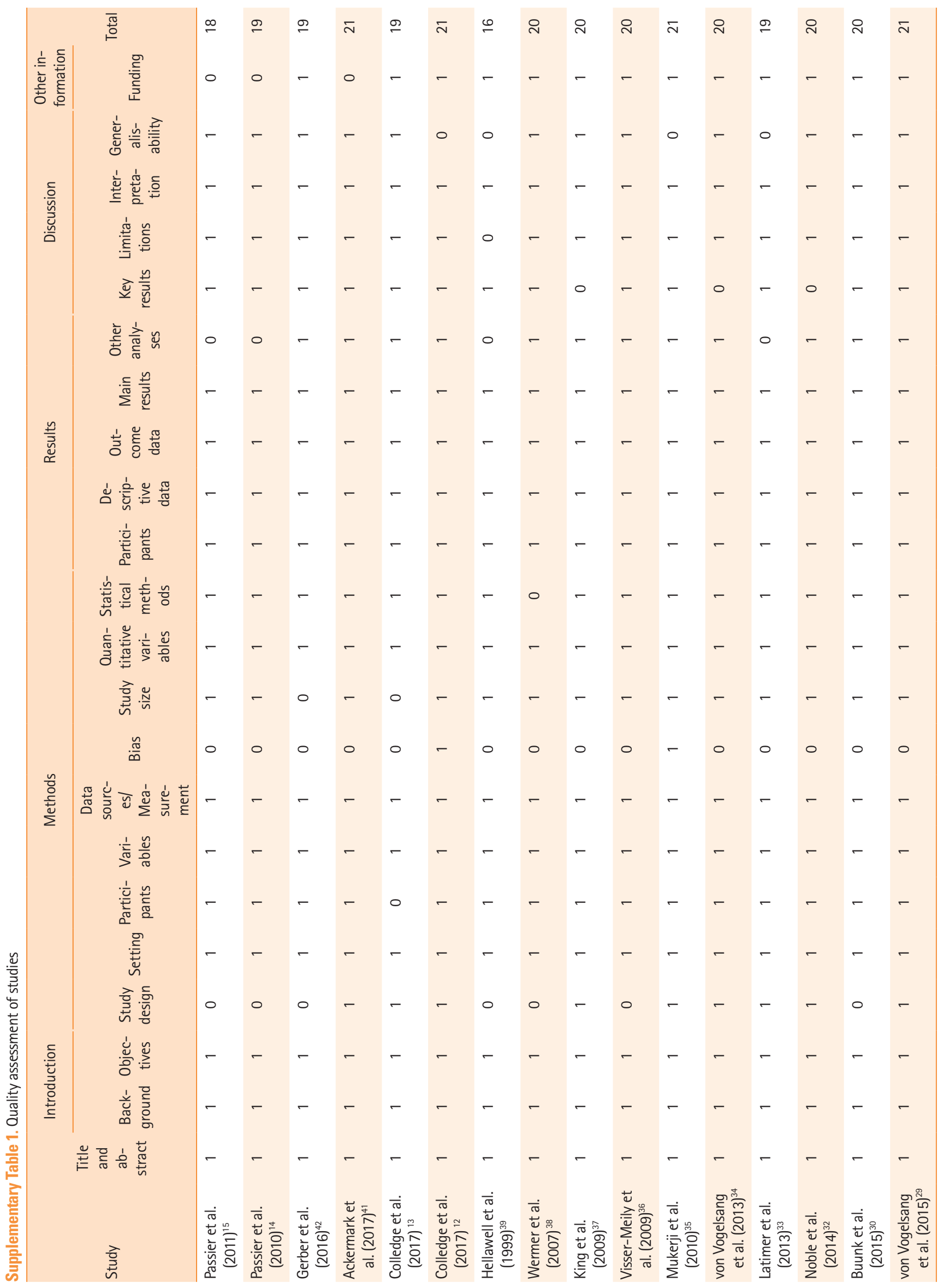




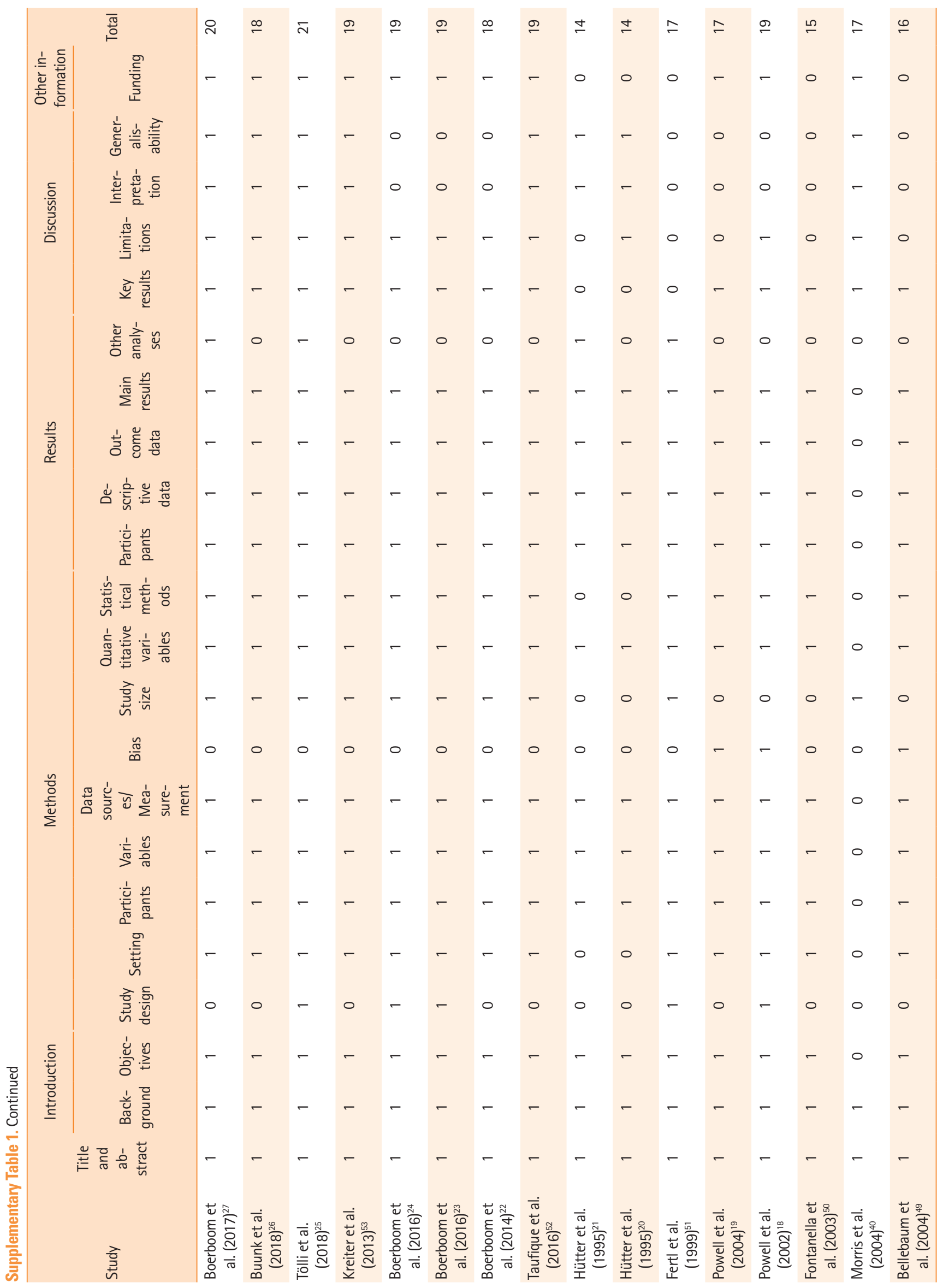




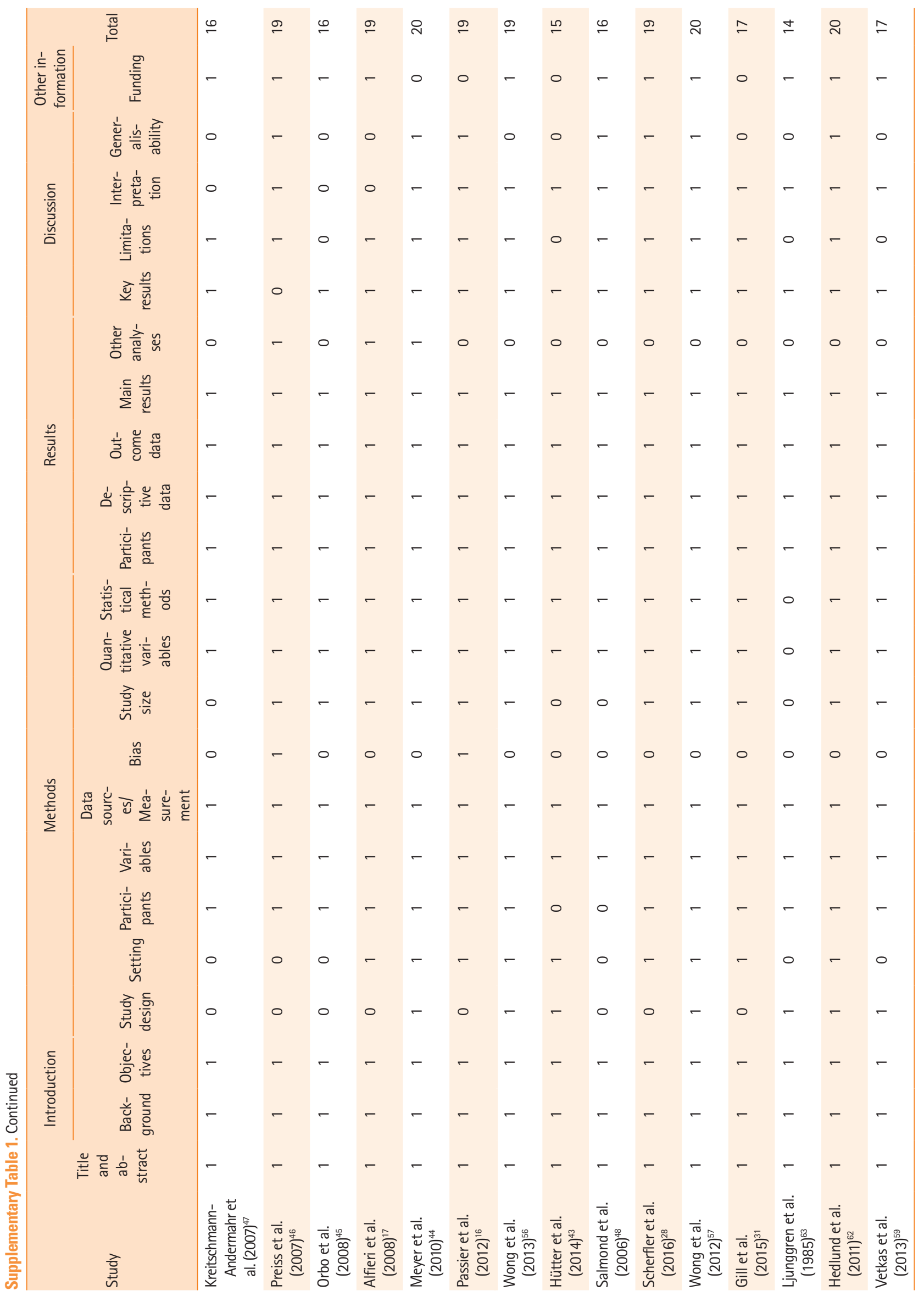




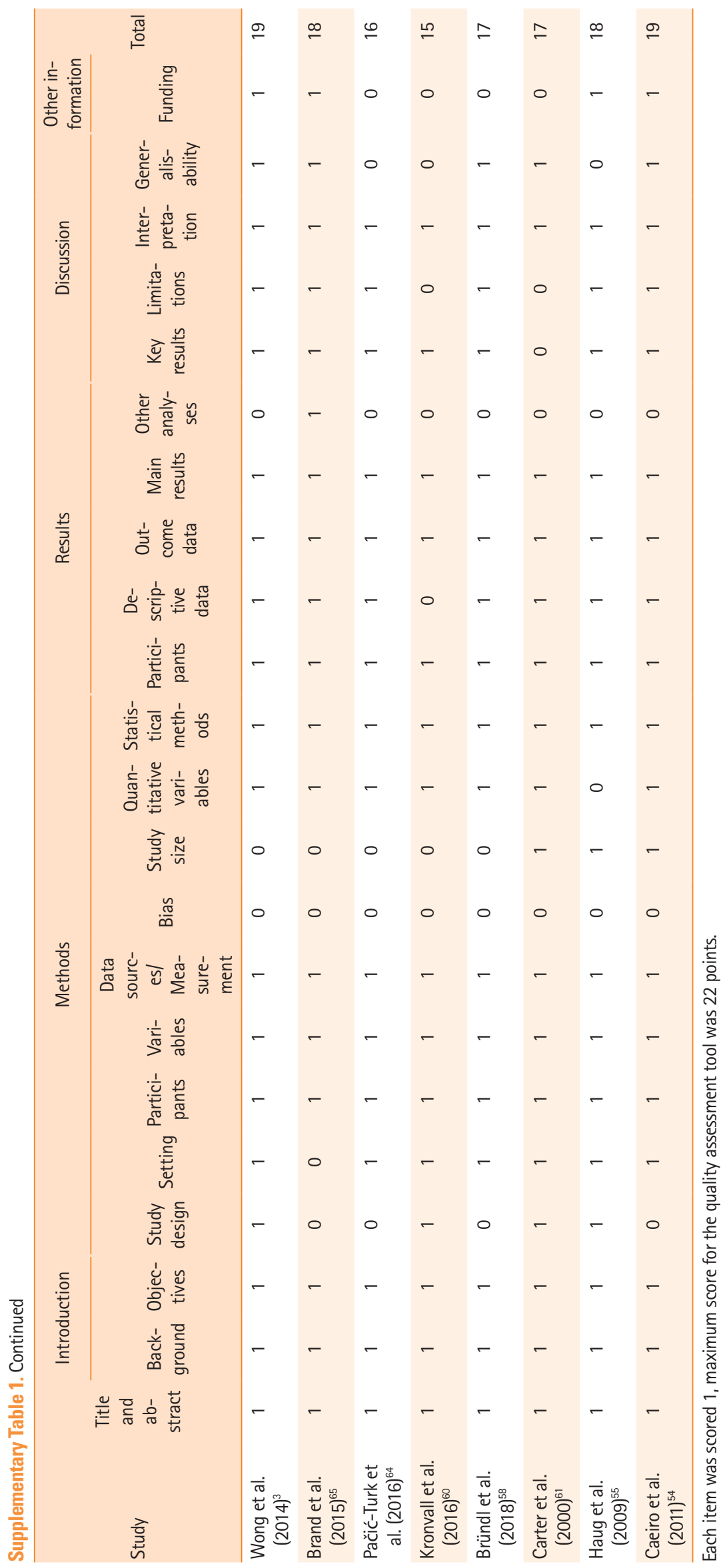


Supplementary Table 2. Severity of depression after subarachnoid hemorrhage

\begin{tabular}{|c|c|c|c|}
\hline Study & Timing after SAH & Measurement & $\begin{array}{l}\text { Depressive symptom } \\
\text { scores (mean } \pm S D)\end{array}$ \\
\hline \multicolumn{4}{|l|}{ Studies using BDI } \\
\hline Alfieri et al. (2008) $)^{17}$ & $\begin{array}{l}\text { On admission (T1) } \\
1 \mathrm{mo}(\mathrm{T} 2) \\
1 \mathrm{yr}(\mathrm{T} 3) \\
3 \mathrm{yr}(\mathrm{T} 4) \\
5 \mathrm{yr}(\mathrm{T} 5)\end{array}$ & $\mathrm{BDI}$ & $\begin{array}{l}\text { T1: } 17.1 \pm 6.5 \\
\text { T2: } 22.1 \pm 3.6 \\
\text { T3: } 19.9 \pm 4.8 \\
\text { T4: } 14.3 \pm 3.8 \\
\text { T5: } 13.2 \pm 3.8\end{array}$ \\
\hline Passier et al. (2010) ${ }^{14}$, Passier et al. $(2011)^{15}$, and Passier et al. (2012) ${ }^{16}$ & $3 \mathrm{mo}$ & $\mathrm{BDI}$ & $9.6 \pm 6.9$ \\
\hline Powell et al. (2002) $)^{18}$ and Powell et al. (2004) ${ }^{19}$ & $\begin{array}{l}3 \mathrm{mo}(\mathrm{T} 1) \\
9 \mathrm{mo}(\mathrm{T} 2) \\
18 \mathrm{mo}(\mathrm{T} 3)\end{array}$ & $\mathrm{BDI}$ & $\begin{array}{l}\text { T1: } 9.6 \pm 6.2 \\
\text { T2: } 9.2 \pm 6.9 \\
\text { T3: } 9.4 \pm 7.3\end{array}$ \\
\hline Ackermark et al. $(2017)^{41}$ & $\begin{array}{l}3 \mathrm{mo}(\mathrm{T} 1) \\
1 \mathrm{yr}(\mathrm{T} 2) \\
2-5 \mathrm{yr}(\mathrm{T} 3)\end{array}$ & $\mathrm{BDI}$ & $\begin{array}{l}\text { T1: } 8.9 \pm 7.0 \\
\text { T2: } 9.3 \pm 7.1 \\
\text { T3: } 11.2 \pm 8.0\end{array}$ \\
\hline Fontanella et al. $(2003)^{50}$ & $6 \mathrm{mo}$ & $\mathrm{BDI}$ & 13.8 (SD NR) \\
\hline Orbo et al. (2008) $)^{45}$ & $1 \mathrm{yr}$ & $\mathrm{BDI}$ & 6 (SD NR) \\
\hline Preiss et al. $(2007)^{46}$ & $1 \mathrm{yr}$ & $\mathrm{BDI}$ & 9.4 \\
\hline Kreitschmann-Andermahr et al. $(2007)^{47}$ & Average 27.3 mo (range, 12-66) & $\mathrm{BDI}$ & $8.33 \pm 5.85$ \\
\hline Colledge et al. (2017) $)^{12,13}$ and Gerber et al. (2016) ${ }^{42}$ & $44 \mathrm{mo}$ & $\mathrm{BDI}$ & $8.9 \pm 6.6$ \\
\hline Salmond et al. $2006^{48}$ & Average 68 mo (range, 14-99) & $\mathrm{BDI}$ & $6.5 \pm 1.4$ \\
\hline \multicolumn{4}{|l|}{ Studies using HADS } \\
\hline von Vogelsang et al. $(2015)^{29}$ & $\begin{array}{l}6 \mathrm{mo}(\mathrm{T} 1) \\
1 \mathrm{yr}(\mathrm{T} 2) \\
2 \mathrm{yr}(\mathrm{T} 3)\end{array}$ & HADS & $\begin{array}{l}\text { T1: } 5.0 \\
\text { T2: } 4.0 \\
\text { T3: } 5.0 \text { (median) }\end{array}$ \\
\hline Scherfler et al. $(2016)^{28}$ & $1 \mathrm{yr}$ & HADS & 1 (median) \\
\hline Visser-Meily et al. (2009) ${ }^{36}$ & Average 3 yr (range, 2-4) & HADS & $4.8 \pm 3.9$ \\
\hline Latimer et al. $(2013)^{33}$ & $40-45 \mathrm{mo}$ & HADS & 6.7 \\
\hline Buunk et al., $2015^{30}$ & Average 4.6 yr (range, 2-10) & HADS & $4.2 \pm 4.3$ \\
\hline Boerboom et al. $(2017)^{27}$ & Average 4.7 yr (range, NR) & HADS & 3.5 (SD NR) \\
\hline Wermer et al. $(2007)^{38}$ & Average 8.9 yr (range, 2.3-18.8) & HADS & $6.2 \pm 3.1$ \\
\hline von Vogelsang et al. $(2013)^{34}$ & Average 10.1 yr (range, 8.8-12) & HADS & 4.0 (median) \\
\hline King et al. (2009) $)^{37}$ & NR & HADS & $4.8 \pm 3.4$ \\
\hline \multicolumn{4}{|l|}{ Studies using other scales } \\
\hline Boerboom et al. (2014) $)^{22}$ and Boerboom et al. (2016) & $\begin{array}{l}0.4 \mathrm{yr}(\mathrm{T} 1) \\
3.9 \mathrm{yr}(\mathrm{T} 2)\end{array}$ & CESD & $\begin{array}{l}\mathrm{T} 1: 13.7 \pm 1.2 \\
\mathrm{~T} 2: 11.9 \pm 1.2\end{array}$ \\
\hline Caeiro et al. $(2011)^{54}$ & $\leq 4$ day & MADRS & $9.2 \pm 7.3$ \\
\hline Haug et al. (2009) ${ }^{55}$ & $1 \mathrm{yr}$ & MADRS & 5.5 (SD NR) \\
\hline Wong et al. $(2012)^{57}$ & $3 \mathrm{mo}$ & GDS & 7 (median) \\
\hline Vetkas et al. $(2013)^{59}$ & Average $4.5 \mathrm{yr}$ (range, 1-10) & GDS & $8.4 \pm 6.9$ \\
\hline Carter et al. $(2000)^{61}$ & Average NR (range, 1-5 yr) & ZDS & 45.6 (SD NR) \\
\hline Brand et al. $(2015)^{65}$ & $5-9 \mathrm{mo}$ & Depression Skala & $1.42 \pm 0.29$ \\
\hline Pačić-Turk et al. (2016) ${ }^{64}$ & $\begin{array}{l}11 \mathrm{mo}(\mathrm{T} 1) \\
12-48 \mathrm{mo}(\mathrm{T} 2)\end{array}$ & $\begin{array}{l}\text { Cornell personality } \\
\text { questionnaire }\end{array}$ & $\begin{array}{l}\mathrm{T} 1: 1.93 \\
\mathrm{~T} 2: 2.65\end{array}$ \\
\hline Kronvall et al. $(2016)^{60}$ & $\begin{array}{l}3-6 \text { mo (T1) } \\
6-12 \text { mo (T2) } \\
12-24 \text { mo (T3) }\end{array}$ & $\begin{array}{l}\text { Psychological General } \\
\text { Well-Being }\end{array}$ & $\begin{array}{l}\text { T1: } 15.0 \pm 3.5 \\
\text { T2: } 15.3 \pm 2.9 \\
\text { T3: } 15.8 \pm 2.9\end{array}$ \\
\hline
\end{tabular}

SAH, subarachnoid hemorrhage; SD, standard deviation; BDI, Beck Depression Inventory; NR, not reported; HADS, Hospital Anxiety Depression Scale; CESD, Center for Epidemiologic Studies Depression; MADRS, Montgomery Åsberg Depression Rating Scale; GDS, Geriatric Depression Scale; ZDS, Zung Depression Scale. 
Supplementary Table 3. Time course of depression after subarachnoid hemorrhage

\begin{tabular}{|c|c|c|}
\hline Study & Timing after SAH & Findings \\
\hline Meyer et al. (2010) $)^{44}$ & $\begin{array}{l}\text { Upon discharge, } \\
6 \text { and } 12 \text { mo }\end{array}$ & $\begin{array}{l}\text { The proportion of patients with depressive symptoms }(\mathrm{BDI}>9) \text { increased from } 24.8 \% \text { at discharge to } \\
61.7 \% \text { at the } 12 \text {-mo follow-up }(P<0.001) \text {. }\end{array}$ \\
\hline Pačić-Turk et al. (2016) ${ }^{64}$ & $11 \mathrm{mo}$ and $12-48 \mathrm{mo}$ & Depressive symptom score increased with time $(\mathrm{t}=-2.417, P=0.019)$. \\
\hline Ackermark et al. (2017) ${ }^{41}$ & $3 \mathrm{mo}, 1 \mathrm{yr}, 2-5 \mathrm{yr}$ & Of patients with depressive symptoms at $3 \mathrm{mo}, 72 \%$ still had symptoms at $2-5 \mathrm{yr}$. \\
\hline Tölli et al. (2018) $)^{25}$ & 3,6 , and $12 \mathrm{mo}$ & No difference in HADS depression scores between $3,6,12$ mo follow-up \\
\hline Boerboom e al. $(2016)^{24}$ & 0.4 and $3.9 \mathrm{yr}$ & Depressive symptoms remained relative stable over time. \\
\hline $\begin{array}{l}\text { Powell et al. }(2002)^{18} \text { and } \\
\text { Powell et al. }(2004)^{19}\end{array}$ & 3,8 and $18 \mathrm{mo}$ & No change in depressive symptoms from 3-9 mo, and from 9-18 mo \\
\hline von Vogelsang et al. (2015) $)^{29}$ & $6 \mathrm{mo}, 1$ and $2 \mathrm{yr}$ & No significant change in depressive symptoms during the 2-year observation period \\
\hline
\end{tabular}

SAH, subarachnoid hemorrhage; BDI, Beck Depression Inventory; HADS, Hospital Anxiety Depression Scale. 